\title{
ON MODELS OF THE BRAID ARRANGEMENT AND THEIR HIDDEN SYMMETRIES
}

\author{
FILIPPO CALLEGARO, GIOVANNI GAIFFI
}

\begin{abstract}
The De Concini-Procesi wonderful models of the braid arrangement of type $A_{n-1}$ are equipped with a natural $S_{n}$ action, but only the minimal model admits an 'hidden' symmetry, i.e. an action of $S_{n+1}$ that comes from its moduli space interpretation. In this paper we explain why the non minimal models don't admit this extended action: they are 'too small'. In particular we construct a supermaximal model which is the smallest model that can be projected onto the maximal model and again admits an extended $S_{n+1}$ action. We give an explicit description of a basis for the integer cohomology of this supermaximal model.

Furthermore, we deal with another hidden extended action of the symmetric group: we observe that the symmetric group $S_{n+k}$ acts by permutation on the set of $k$-codimensionl strata of the minimal model. Even if this happens at a purely combinatorial level, it gives rise to an interesting permutation action on the elements of a basis of the integer cohomology.
\end{abstract}

\section{INTRODUCTION}

In this paper we focus on two different 'hidden' extended actions of the symmetric group on wonderful models of the (real or complexified) braid arrangement. As it is well known, there are several De Concini-Procesi models associated with the arrangement of type $A_{n-1}$ (see [4], [5]); these are smooth varieties, proper over the complement of the arrangement, in which the union of the subspaces is replaced by a divisor with normal crossings. Among these spaces there is a minimal one (i.e. there are birational projections from the other spaces onto it), and a maximal one (i.e. there are birational projections from it onto the other spaces). The natural $S_{n}$ action on the complement of the arrangement of type $A_{n-1}$ extends to all of these models.

The first of the two extended actions which we deal with is well known and comes from the following remark: the minimal projective (real or complex) De Concini-Procesi model of type $A_{n-1}$ is isomorphic to the moduli space $\bar{M}_{0, n+1}$ of $n+1$-pointed stable curves of genus 0 , therefore it carries an 'hidden' extended action of $S_{n+1}$ that has been studied by several authors (see for instance [23, 35], 11]).

Now we observe that the $S_{n+1}$ action cannot be extended to the non-minimal models (we show this by an example in Section 5 ).

Why does this happen? This is the first problem discussed in the present paper. We answer to this question by showing in Section 6 that the maximal model is, in a sense, 'too small'. This takes two steps (see Theorem 6.1):

Date: October 28, 2018. 
(1) we identify in a natural way its strata with a subset $\mathcal{T}$ of 1-codimensional strata of a 'supermaximal' model on which the $S_{n+1}$ action is defined. This supermaximal model is obtained by blowing up some strata in the maximal model, but it also belongs to the family $\mathcal{L}$ of models obtained by blowing up building sets of strata in the minimal model; in fact it is the model obtained by blowing up all the strata of the minimal model. The models in $\mathcal{L}$ are examples of some well known constructions that, starting from a 'good' stratified variety, produce models by blowing up a suitable subset of strata (see 30], 29], 19] and also 9] for further references);

(2) we show that the closure of $\mathcal{T}$ under the $S_{n+1}$ action is the set of all the strata of the supermaximal model. More precisely, this means that the supermaximal model is the minimal model in $\mathcal{L}$ that admits a birational projection onto the maximal model and is equipped with the $S_{n+1}$ action.

The second problem addressed by this paper is the computation of the integer cohomology of the complex supermaximal modes described above. The cohomology module provides 'geometric' extended representations of $S_{n+1}$ and in Theorem 7.2 we exhibit an explicit basis for it. Actually, the statement of Theorem 7.2 is much more general: given any complex subspace arrangement we consider its minimal De Concini-Procesi model and we describe a basis for the integer cohomology of the variety obtained by blowing up all the strata in this minimal model (this variety generalizes in a way the notion of supermaximal model).

We then compute a generating formula for the Poincaré polynomials of the complex supermaximal models of braid arrangements (see Theorems 8.1, 8.2). As a consequence, we also give a formula for the Euler characteristic series in the real case, where Euler secant numbers appear (see Corollary 8.3.

In the last two sections, Section 9 and Section 10, we show that there is another hidden extended action of the symmetric group on the minimal model of a braid arrangement, that is different from the action described above.

In fact, motivated by a combinatorial remark proven in 20 , we observe that the symmetric group $S_{n+k}$ acts by permutation on the set of $k$-codimensionl strata of the minimal model of type $A_{n-1}$.

This happens at a purely combinatorial level and it does not correspond to a geometric action on the minimal model, nevertheless it gives rise to an interesting permutation action on the elements of a basis of the integer cohomology of the complex minimal model. The splitting of these elements into orbits allows us to write (see Theorem 10.1) a generating formula for the Poincaré polynomials of the complex minimal models that is different from the ones available in the literature (see for instance the recursive formula for the Poincaré series computed, in three different ways, in [31], [40], [17]).

\section{Wonderful Models}

2.1. The Geometric Definition of Building Sets and Nested Sets. In this section we recall from [4], [5] the definitions of building set and nested set of subspaces. Let $V$ be a real or complex finite dimensional vector space endowed with an Euclidean or Hermitian non-degenerate product and let $\mathcal{A}$ be a central subspace arrangement in $V$. For every $A \in V$, we will denote by $A^{\perp}$ its orthogonal. We denote by $\mathcal{C}_{\mathcal{A}}$ the closure under the sum of $\mathcal{A}$ and by $\mathcal{A}^{\perp}$ the arrangement of subspaces in 
$V$

$$
\mathcal{A}^{\perp}=\left\{A^{\perp} \mid A \in \mathcal{A}\right\} .
$$

Definition 2.1. The collection of subspaces $\mathcal{G} \subset \mathcal{C}_{\mathcal{A}}$ is called building set associated to $\mathcal{A}$ if $\mathcal{C}_{\mathcal{A}}=\mathcal{C}_{\mathcal{G}}$ and every element $C$ of $\mathcal{C}_{\mathcal{A}}$ is the direct sum $C=G_{1} \oplus G_{2} \oplus \ldots \oplus G_{k}$ of the maximal elements $G_{1}, G_{2}, \ldots, G_{k}$ of $\mathcal{G}$ contained in $C$ (this is called the $\mathcal{G}$ decomposition of $C$ ).

Given a subspace arrangement $\mathcal{A}$, there are several building sets associated to it. Among these there always are a maximum and a minimum (with respect to inclusion). The maximum is $\mathcal{C}_{\mathcal{A}}$, the minimum is the building set of irreducibles that is defined as follows.

Definition 2.2. Given a subspace $U \in \mathcal{C}_{\mathcal{A}}$, a decomposition of $U$ in $\mathcal{C}_{\mathcal{A}}$ is a collection $\left\{U_{1}, \ldots, U_{k}\right\}(k>1)$ of non zero subspaces in $\mathcal{C}_{\mathcal{A}}$ such that

(1) $U=U_{1} \oplus \cdots \oplus U_{k}$;

(2) for every subspace $A \in \mathcal{C}_{\mathcal{A}}$ such that $A \subset U$, we have $A \cap U_{1}, \ldots, A \cap U_{k} \in \mathcal{C}_{\mathcal{A}}$ and $A=\left(A \cap U_{1}\right) \oplus \cdots \oplus\left(A \cap U_{k}\right)$.

Definition 2.3. A nonzero subspace $F \in \mathcal{C}_{\mathcal{A}}$ which does not admit a decomposition is called irreducible and the set of irreducible subspaces is denoted by $\mathcal{F}_{\mathcal{A}}$.

Remark 2.1. As an example, let us consider a root system $\Phi$ in $V$ (real or complexified vector space) and its associated root arrangement (i.e. $\mathcal{A}^{\perp}$ is the hyperplane arrangement provided by the hyperplanes orthogonal to the roots in $\Phi)$. In this case the building set of irreducibles is the set whose elements are the subspaces spanned by the irreducible root subsystems of $\Phi$ (see [40]).

Definition 2.4. Let $\mathcal{G}$ be a building set associated to $\mathcal{A}$. A subset $\mathcal{S} \subset \mathcal{G}$ is called $\left(\mathcal{G}\right.$-)nested, if given any subset $\left\{U_{1}, \ldots, U_{h}\right\} \subseteq \mathcal{S}$ (with $h>1$ ) of pairwise non comparable elements, we have that $U_{1}+\cdots+U_{h} \notin \mathcal{G}$.

2.2. The Example of the Root System $A_{n-1}$. Let $V=\mathbb{R}^{n}$ or $\mathbb{C}^{n}$ and let us consider the real or complexified root arrangement of type $A_{n-1}$. We think of it as an essential arrangement, i.e. we consider the hyperplanes defined by the equations $x_{i}-x_{j}=0$ in the quotient space $\left.V /<(1,1, \ldots, 1)\right\rangle$.

Let us denote by $\mathcal{F}_{A_{n-1}}$ the building set of irreducibles associated to this arrangement. According to Remark 2.1, it is made by all the subspaces in $V$ spanned by the irreducible root subsystems. Therefore there is a bijective correspondence between the elements of $\mathcal{F}_{A_{n-1}}$ and the subsets of $\{1, \cdots, n\}$ of cardinality at least two: if the orthogonal of $A \in \mathcal{F}_{A_{n-1}}$ is the subspace described by the equation $x_{i_{1}}=x_{i_{2}}=\cdots=x_{i_{k}}$ then we represent $A$ by the set $\left\{i_{1}, i_{2}, \ldots, i_{k}\right\}$. As a consequence, a $\mathcal{F}_{A_{n-1}}$-nested set $\mathcal{S}$ is represented by a set (which we still call $\mathcal{S}$ ) of subsets of $\{1, \cdots, n\}$ with the property that any of its elements has cardinality $\geq 2$ and if $I$ and $J$ belong to $\mathcal{S}$ than either $I \cap J=\emptyset$ or one of the two sets is included into the other.

As an example we can consider the $\mathcal{F}_{A_{8}}$-nested set represented by the three sets $\{1,5\},\{2,4,6\},\{2,4,6,7,8\}$. This means that the elements of the nested set are the three subspaces whose orthogonal subspaces in $V /<(1,1, \ldots, 1)\rangle$ are described respectively by the equations $x_{1}=x_{5}, x_{2}=x_{4}=x_{6}$ and $x_{2}=x_{4}=x_{6}=x_{7}=x_{8}$.

We observe that we can represent a $\mathcal{F}_{A_{n-1}}$-nested set $\mathcal{S}$ by an oriented forest on $n$ leaves in the following way. We consider the set $\tilde{\mathcal{S}}=\mathcal{S} \cup\{1\} \cup\{2\} \cup \cdots \cup\{n\}$. Then 
the forest coincides with the Hasse diagram of $\tilde{\mathcal{S}}$ viewed as a poset by the inclusion relation: the roots of the trees correspond to the maximal elements of $\mathcal{S}$, and the orientation goes from the roots to the leaves, that are the vertices $\{1\},\{2\}, \ldots,\{n\}$.

Let us now focus on the maximal building set $\mathcal{C}_{A_{n-1}}$ associated with the root arrangement of type $A_{n-1}$. It is made by all the subspaces that can be obtained as the span of a set of roots. Using the same notation as before, these subspaces can be put in bijective correspondence with the partitions of $\{1, \cdots, n\}$ such that at least one part has cardinality $\geq 2$.

For instance,

$$
\{1,4\},\{2\},\{3,5,9\},\{6\},\{7,8\}
$$

corresponds to the subspace whose orthogonal is described by the equations $x_{1}=x_{4}$ and $x_{3}=x_{5}=x_{9}$ and $x_{7}=x_{8}$.

The $\mathcal{C}_{A_{n-1}}$-nested sets are given by chains of subspaces in $\mathcal{C}_{A_{n-1}}$ (with respect to inclusion). In terms of partitions, this corresponds to give chains of the above described partitions of $\{1, \cdots, n\}$ (with respect to the refinement relation).

One can find in 21 a description of the maximal model $Y_{\mathcal{C}_{A_{n-1}}}$ and in 22 a description of all the $S_{n}$-invariant building sets associated with the root system $A_{n-1}$.

2.3. The construction of wonderful models and their cohomology. In this section we recall from 4 the construction and the main properties of the De Concini-Procesi models.

The interest in these models was at first motivated by an approach to Drinfeld's construction of special solutions for Khniznik-Zamolodchikov equation (see 10]). Moreover, in [4 it was shown, using the cohomology description of these models, that the rational homotopy type of the complement of a complex subspace arrangement depends only on the intersection lattice.

Then real and complex De Concini-Procesi models turned out to play a key role in several fields of mathematical research: subspace and toric arrangements, toric varieties (see for instance [7], 15], 36]), tropical geometry (see [14), moduli spaces and configuration spaces (see for instance [1], 28]), box splines, vector partition functions and index theory (see [6], [2]), discrete geometry (see [12]).

Let us recall how they are defined. We will focus on the case when $\mathbb{K}=\mathbb{R}$ or $\mathbb{K}=\mathbb{C}$. Let $\mathcal{A}$ be a subspace arrangement in the real or complex space $V$ endowed with a non-degenerate euclidean or Hermitian product and let $\mathcal{M}\left(\mathcal{A}^{\perp}\right)$ be the complement in $V$ of the arrangement $\mathcal{A}^{\perp}$. Let $\mathcal{G}$ be a building set associated to $\mathcal{A}$ (we can suppose that it contains $V$ ). Then one considers the map

$$
i: \mathbb{P}\left(\mathcal{M}\left(\mathcal{A}^{\perp}\right)\right) \rightarrow \mathbb{P}(V) \times \prod_{D \in \mathcal{G}-\{V\}} \mathbb{P}\left(V / D^{\perp}\right)
$$

where in the first coordinate we have the inclusion and the map from $\mathcal{M}\left(\mathcal{A}^{\perp}\right)$ to $\mathbb{P}\left(V / D^{\perp}\right)$ is the restriction of the canonical projection $\left(V-D^{\perp}\right) \rightarrow \mathbb{P}\left(V / D^{\perp}\right)$.

Definition 2.5. The (compact) wonderful model $Y_{\mathcal{G}}$ is obtained by taking the closure of the image of $i$.

De Concini and Procesi in 4] proved that the complement $\mathcal{D}$ of $\mathbb{P}\left(\mathcal{M}\left(\mathcal{A}^{\perp}\right)\right)$ in $Y_{\mathcal{G}}$ is a divisor with normal crossings whose irreducible components are in bijective correspondence with the elements of $\mathcal{G}-\{V\}$ and are denoted by $\mathcal{D}_{G}(G \in \mathcal{G}-\{V\})$. 
If we denote by $\pi$ the projection of $Y_{\mathcal{G}}$ onto the first component $\mathbb{P}(V)$, then $\mathcal{D}_{G}$ can be characterized as the unique irreducible component such that $\pi\left(\mathcal{D}_{G}\right)=$ $\mathbb{P}\left(G^{\perp}\right)$.

A complete characterization of the boundary is then provided by the observation that, if we consider a collection $\mathcal{T}$ of subspaces in $\mathcal{G}$ containing $V$, then

$$
\mathcal{D}_{\mathcal{T}}=\bigcap_{A \in \mathcal{T}-\{V\}} \mathcal{D}_{A}
$$

is non empty if and only if $\mathcal{T}$ is $\mathcal{G}$-nested, and in this case $\mathcal{D}_{\mathcal{T}}$ is a smooth irreducible subvariety obtained as a normal crossing intersection. Sometimes we will denote $Y_{\mathcal{G}}$ by $\mathcal{D}_{\mathcal{S}}$ with $\mathcal{S}=\{V\}$.

The integer cohomology ring of the models $Y_{\mathcal{G}}$ in the complex case was studied in 4], where a presentation by generators and relations was provided. The cohomology is torsion free, and in 40] Yuzvinski explicitly described some $\mathbb{Z}$-bases (see also [17]). We briefly recall these results (for a description of the cohomology ring in the real case see [36]).

Let $\mathcal{G}$ be a building set containing $V$ and let us consider a $\mathcal{G}$-nested set $\mathcal{S}$. We take a subset $\mathcal{H} \subset \mathcal{G}$ and an element $B \in \mathcal{G}$ with the property that $A \subsetneq B$ for all $A \in \mathcal{H}$ and we put $\mathcal{S}_{B}=\{A \in \mathcal{S}: A \subsetneq B\}$. As in [4], we define the non negative integer $d_{\mathcal{H}, B}^{\mathcal{S}}$ :

\section{Definition 2.6.}

$$
d_{\mathcal{H}, B}^{\mathcal{S}}=\operatorname{dim} B-\operatorname{dim}\left(\sum_{A \in \mathcal{H} \cup \mathcal{S}_{B}} A\right)
$$

Then we consider the polynomial ring $\mathbb{Z}\left[c_{A}\right]$ where the variables $c_{A}$ are indexed by the elements of $\mathcal{G}$.

Definition 2.7. Given $\mathcal{G}, \mathcal{S}, \mathcal{H}$ and $B$ as before, we define the following polynomial in $\mathbb{Z}\left[c_{A}\right]$ :

$$
P_{\mathcal{H}, B}^{\mathcal{S}}=\left(\prod_{A \in \mathcal{H}} c_{A}\right)\left(\sum_{B \subset C} c_{C}\right)^{d_{\mathcal{H}, B}^{\mathcal{S}}}
$$

Let us now call by $I_{\mathcal{S}}$ the ideal in $\mathbb{Z}\left[c_{A}\right]$ generated by these polynomials, for fixed $\mathcal{S}$ and varying $\mathcal{H}, B$.

Theorem 2.1 (see 4, Section 5.2]). Let $\mathcal{G}$ and $\mathcal{S}$ be as before, and let us consider the complex model $Y_{\mathcal{G}}$. The natural map $\phi: \mathbb{Z}\left[c_{A}\right] \mapsto H^{*}\left(\mathcal{D}_{\mathcal{S}}, \mathbb{Z}\right)$, defined by sending $c_{A}$ to the cohomology class $\left[\mathcal{D}_{A}\right]$ associated to the divisor $\mathcal{D}_{A}$ (restricted to $\mathcal{D}_{S}$ ), induces an isomorphism between $\mathbb{Z}\left[c_{A}\right] / I_{\mathcal{S}}$ and $H^{*}\left(\mathcal{D}_{\mathcal{S}}, \mathbb{Z}\right)$. In particular, in the case $\mathcal{S}=\{V\}$, we obtain

$$
\mathbb{Z}\left[c_{A}\right] / I_{\{V\}} \simeq H^{*}\left(Y_{\mathcal{G}}, \mathbb{Z}\right)
$$

Definition 2.8. Let $\mathcal{G}$ and $\mathcal{S}$ be as before. A function $f: \mathcal{G} \mapsto \mathbb{N}$ is called $\mathcal{G}, \mathcal{S}$-admissible if it is $f=0$ or if $f \neq 0$, supp $f \cup \mathcal{S}$ is $\mathcal{G}$-nested and, for every $A \in \operatorname{supp} f, f(A)<d_{(\operatorname{supp} f)_{A}, A}^{\mathcal{S}}$.

Now, given a $\mathcal{G}, \mathcal{S}$-admissible function $f$, we can consider in $H^{*}\left(\mathcal{D}_{\mathcal{S}}, \mathbb{Z}\right) \simeq \mathbb{Z}\left[c_{A}\right] / I_{\mathcal{S}}$ the monomial $m_{f}=\prod_{A \in \mathcal{G}} c_{A}^{f(A)}$. We will call " $\mathcal{G}, \mathcal{S}$-admissible" such monomials. 
Theorem 2.2 (see [40, Section 3] and [17, Section 2]). The set $\mathcal{B}_{\mathcal{G}, \mathcal{S}}$ of $\mathcal{G}, \mathcal{S}$ admissible monomials is a $\mathbb{Z}$-basis for $H^{*}\left(\overline{\mathcal{D}_{\mathcal{S}}}, \mathbb{Z}\right)$.

2.4. A More General Construction. The construction of De Concini-Procesi models can be viewed as a special case of other more general constructions that, starting from a 'good' stratified variety, produce models by blowing up a suitable subset of strata. Among these there are the models described by MacPherson and Procesi in 30 and by Li in 29. In Li's paper one can also find a comparison among several constructions of wonderful compactifications by Fulton-Machperson ([16]), Ulyanov ([38]), Kuperberg-Thurston ([27]), Hu ([24]). A further interesting survey including tropical compactifications can be found in Denham's paper 9].

We recall here some basic facts adopting the language and the notation of Li's paper.

Definition 2.9. A simple arrangement of subvarieties (or 'simple stratification') of a nonsingular variety $Y$ is a finite set $\Lambda=\left\{\Lambda_{i}\right\}$ of nonsingular closed subvarieties $\Lambda_{i}$ properly contained in $Y$ satisfying the following conditions:

(i) the intersection of $\Lambda_{i}$ and $\Lambda_{j}$ is nonsingular and the tangent bundles satisfy $T\left(\Lambda_{i} \cap \Lambda_{j}\right)=T\left(\Lambda_{i}\right)_{\mid\left(\Lambda_{i} \cap \Lambda_{j}\right)} \cap T\left(\Lambda_{j}\right)_{\mid\left(\Lambda_{i} \cap \Lambda_{j}\right)}$,

(ii) $\Lambda_{i} \cap \Lambda_{j}$ either is equal to some stratum in $\Lambda$ or is empty.

Definition 2.10. Let $\Lambda$ be an arrangement of subvarieties of $Y$. A subset $\mathcal{G}^{\prime} \subseteq \Lambda$ is called a building set of $\Lambda$ if $\forall \Lambda_{i} \in \Lambda-\mathcal{G}^{\prime}$ the minimal elements in $\left\{G \in \mathcal{G}^{\prime}: G \supseteq \Lambda_{i}\right\}$ intersect transversally and the intersection is $\Lambda_{i}$.

Then, if one has a simple stratification $\Lambda$ of a nonsingular variety $Y$ and a building set $\mathcal{G}^{\prime}$, one can construct a wonderful model $Y_{\mathcal{G}^{\prime}}$ by considering (by analogy with 4]) the closure of the image of the locally closed embedding

$$
\left(Y-\bigcup_{\Lambda_{i} \in \Lambda} \Lambda_{i}\right) \rightarrow \prod_{G \in \mathcal{G}^{\prime}} B l_{G} Y
$$

where $B l_{G} Y$ is the blowup of $Y$ along $G$.

It turns out that

Theorem 2.3 (see 29, Theorem 1.3]). If one arranges the elements $G_{1}, G_{2}, \ldots, G_{N}$ of $\mathcal{G}^{\prime}$ in such a way that for every $1 \leq i \leq N$ the set $\left\{G_{1}, G_{2}, \ldots, G_{i}\right\}$ is building, then $Y_{\mathcal{G}^{\prime}}$ is isomorphic to the variety

$$
B l_{\widetilde{G}_{N}} B l_{\widetilde{G}_{N-1}} \cdots B l_{\widetilde{G}_{2}} B l_{G_{1}} Y
$$

where $\widetilde{G}_{i}$ denotes the dominant transform of $G_{i}$ in $B l_{\widetilde{G}_{i-1}} \cdots B l_{\widetilde{G}_{2}} B l_{G_{1}} Y$.

Remark 2.2. As remarked by Procesi-MacPherson in [30, Section 2.4] it is always possible to choose a linear ordering on the set $\mathcal{G}^{\prime}$ such that every initial segment is building. We can do this by ordering $\mathcal{G}^{\prime}$ in such a way that we always blow up first the strata of smaller dimension.

We show two examples that will be crucial in the following sections.

Example 2.1. In the case of subspace arrangements, the De Concini-Procesi construction and the above construction produce the same models (the only warning is that in the preceding sections a building set $\mathcal{G}$ was described in a dual way, so the building set of subvarieties $\mathcal{G}^{\prime}$ is made by the orthogonals of the subspaces in $\mathcal{G}$ ). 
Example 2.2. Given a De Concini-Procesi model $Y_{\mathcal{G}}$, we notice that its boundary strata give rise to a simple arrangement of subvarieties, and that the set of all strata is a building set. So it is possibile to obtain a 'model of the model $Y_{\mathcal{G}}$ '. The boundary strata of these 'models of models' are indexed by the nested sets of the building set of all the strata of $Y_{\mathcal{G}}$. More precisely, according to the definition given in [30. Section 4], a nested set $\mathcal{S}$ in this sense is a collection of $\mathcal{G}$-nested sets containing $\{V\}$ linearly ordered by inclusion (we will come back to this, using a more combinatorial definition, in Section 3).

\section{Combinatorial Building Sets}

After De Concini and Procesi's paper 4, nested sets and building sets appeared in the literature, connected with several combinatorial problems. In 13 building sets and nested sets were defined in the general context of meet-semilattices, and in 8 their connection with Dowling lattices was investigated. Other purely combinatorial definitions were used to give rise to the polytopes that were named nestohedra in 33 .

Here we recall the combinatorial definitions of building sets and nested sets of a power set in the spirit of 33], 34] (one can refer to [32, Section 2] for a short comparison among various definitions and notations in the literature).

Definition 3.1. A building set of the power set $\mathcal{P}(\{1,2, \ldots, n\})$ is a subset $\mathcal{B}$ of $\mathcal{P}(\{1,2, \ldots, n\})$ such that:

a) If $A, B \in \mathcal{B}$ have nonempty intersection, then $A \cup B \in \mathcal{B}$.

b) The set $\{i\}$ belongs to $\mathcal{B}$ for every $i \in\{1,2, \ldots, n\}$.

Definition 3.2. A (nonempty) subset $\mathcal{S}$ of a building set $\mathcal{B}$ is a $\mathcal{B}$-nested set (or just nested set if the context is understood) if and only if the following two conditions hold:

a) For any $I, J \in \mathcal{S}$ we have that either $I \subset J$ or $J \subset I$ or $I \cap J=\emptyset$.

b) Given elements $\left\{J_{1}, \ldots, J_{k}\right\}(k \geq 2)$ of $\mathcal{S}$ pairwise not comparable with respect to inclusion, their union is not in $\mathcal{B}$.

Definition 3.3. The nested set complex $\mathcal{N}(\mathcal{B})$ is the poset of all the nested sets of $\mathcal{B}$ ordered by inclusion.

We notice that actually $\mathcal{N}(\mathcal{B}) \cup\{\emptyset\}$ is a simplicial complex.

Definition 3.4. If the set $\mathcal{B}$ has a minimum $\mu$, the nested set complex $\mathcal{N}^{\prime}(\mathcal{B})$ is the poset of all the nested sets of $\mathcal{B}$ containing $\mu$, ordered by inclusion.

In particular, let us denote by $\mathcal{B}(n-1)$ the poset $\mathcal{N}^{\prime}\left(\mathcal{F}_{A_{n-1}}\right)$ given by the nested sets in $\mathcal{F}_{A_{n-1}}$ that contain $\{V\}$.

We observe that any element in $\mathcal{N}^{\prime}\left(\mathcal{F}_{A_{n-1}}\right)$ can be obtained by the union of $\{V\}$ with an element of

$$
\mathcal{P}\left(\mathcal{A}_{1}^{\prime}\right) \cup \mathcal{P}\left(\mathcal{A}_{2}^{\prime}\right) \cup \ldots \cup \mathcal{P}\left(\mathcal{A}_{s}^{\prime}\right)
$$

where $\mathcal{A}_{j}^{\prime}=\mathcal{A}_{j}-\{V\}$ and $\mathcal{A}_{j}$ are all the maximal nested sets associated with the building set $\mathcal{F}_{A_{n-1}}$ (and $\mathcal{P}()$ denotes the power set). Given a simplicial complex $\mathcal{C}$ which is based on some sets $\mathcal{A}_{1}^{\prime}, . ., \mathcal{A}_{s}^{\prime}$ (i.e., it is equal to $\mathcal{P}\left(\mathcal{A}_{1}^{\prime}\right) \cup \mathcal{P}\left(\mathcal{A}_{2}^{\prime}\right) \cup \ldots \cup$ $\mathcal{P}\left(\mathcal{A}_{s}^{\prime}\right)$ ), Feichtner and Kozlov's definition of building set of a meet semilattice (see 13. Section 2]) can be expressed in the following way: $\mathcal{B} \subseteq \mathcal{C}$ is a building set of $\mathcal{C}$ 
if and only if for every $j=1,2, \ldots, s$ the set $\mathcal{B} \cap \mathcal{P}\left(\mathcal{A}_{j}^{\prime}\right)$ is a building set of $\mathcal{P}\left(\mathcal{A}_{j}^{\prime}\right)$ in the sense of Definition 3.1 .

Again, according to Feichtner and Kozlov, given a building set $\mathcal{B}$ of $\mathcal{C}$ as before, a $\mathcal{B}$-nested set is a subset $\mathcal{S}$ of $\mathcal{B}$ such that, for every antichain (with respect to inclusion) $\left\{X_{1}, X_{2}, \ldots, X_{l}\right\} \subseteq \mathcal{S}$, the union $X_{1} \cup X_{2} \cup . . \cup X_{l}$ belongs to $\mathcal{C}-\mathcal{B}$.

These definitions of building set and nested sets can be extended in a natural way to $\mathcal{B}(n-1)=\mathcal{N}^{\prime}\left(\mathcal{F}_{A_{n-1}}\right)$. In particular, the maximal building set of $\mathcal{B}(n-1)$ is $\mathcal{B}(n-1)$ itself. As we observed in Section 2.3 the strata of $Y_{\mathcal{F}_{A_{n-1}}}$ are indexed by the elements of $\mathcal{B}(n-1)$ and, as we remarked in Section 2.4, the set of all these strata is a building set in the sens $\mathrm{f}^{1}$ of $30 \sqrt{29}$ and $[19$, therefore we can construct the corresponding (real or complex) variety $Y_{\mathcal{B}(n-1)}$.

Translating into these combinatorial terms the definition given in 30, Section 4], the strata of the variety $Y_{\mathcal{B}(n-1)}$ are indexed by the nested sets of $\mathcal{B}(n-1)$ containing $\{V\}$ in the following way: a stratum of codimension $r$ is indexed by $\left\{\{V\}, \mathcal{T}_{1}, \ldots, \mathcal{T}_{r}\right\}$ where each $\mathcal{T}_{i}$ belongs to $\mathcal{B}(n-1)$ and

$$
\{V\} \subsetneq \mathcal{T}_{1} \subsetneq \cdots \subsetneq \mathcal{T}_{r} .
$$

\section{The Geometric EXtended $S_{n+1}$ ACTION ON $Y_{\mathcal{F}_{A_{n-1}}}$}

We recall that there is a well know 'extended' $S_{n+1}$ action on the De ConciniProcesi (real or complex) model $Y_{\mathcal{F}_{A_{n-1}}}$ : it comes from the isomorphism with the moduli space $\bar{M}_{0, n+1}$ and the character of the resulting representation on cohomology has been computed in 35] in the real case, and in 23 in the complex case.

In order to describe how $S_{n+1}$ acts on the strata of $Y_{\mathcal{F}_{A_{n-1}}}$ it is sufficient to show the corresponding action on $\mathcal{F}_{A_{n-1}}$, since these strata are indexed by the elements of $\mathcal{B}(n-1)$.

Let $\Delta=\left\{\alpha_{0}, \alpha_{1}, \ldots, \alpha_{n-1}\right\}$ be a basis for the root system $\Phi_{A_{n}}$ of type $A_{n}$ (we added to a basis of $A_{n-1}$ the extra root $\alpha_{0}$ ). We identify in the standard way $S_{n+1}$ with the group which permutes $\{0,1, \ldots, n\}$ and $s_{\alpha_{0}}$ with the transposition $(0,1)$. Therefore $S_{n}$, the subgroup generated by $\left\{s_{\alpha_{1}}, \ldots, s_{\alpha_{n-1}}\right\}$, is identified with the subgroup which permutes $\{1, \ldots, n\}$.

Let $A$ be a subspace in $\mathcal{F}_{A_{n-1}}$ different from $V$, let $\sigma \in S_{n+1}$ and let us consider the subspace $\sigma A$ according to the natural action of $S_{n+1}$ on $\mathcal{F}_{A_{n}}$. Morover we denote by $\bar{A}$ the subspace generated by all the roots of $\Phi_{A_{n}}$ that are orthogonal to $A$. We notice that if some of the roots contained in $\sigma A$ have $\alpha_{0}$ in their support then $\sigma \bar{A}$ belongs to $\mathcal{F}_{A_{n-1}}$. Therefore we define the action of $S_{n+1}$ on $\mathcal{F}_{A_{n-1}}$ as follows. Let $\sigma \in S_{n+1}$ and $A \in \mathcal{F}_{A_{n-1}}$. We set $\sigma \cdot V:=V$ and for $A \neq V$ we define

$$
\sigma \cdot A:= \begin{cases}\sigma A & \text { if } \sigma A \in \mathcal{F}_{A_{n-1}} \\ \sigma \bar{A} & \text { otherwise. }\end{cases}
$$

Let us now write explicitly how the above described action extends to $\mathcal{B}(n-1)$. Let

$$
\mathcal{S}=\left\{V, A_{1}, A_{2}, \ldots, A_{k}, B_{1}, B_{2}, \ldots, B_{s}\right\}
$$

be an element of $\mathcal{B}(n-1)$, i.e. a nested set in $\mathcal{F}_{A_{n-1}}$ that contains $V$ and let $\sigma \in S_{n+1}$. Moreover, let us suppose that, for every subspace $A_{j}$, the subspace

\footnotetext{
${ }^{1}$ According to the original definition, the building set is $\mathcal{B}(n-1)-\{\{V\}\}$, but one can immediately check that one can add $\{V\}$ without affecting the construction.
} 
$\sigma A_{j}$ doesn't belong to $\mathcal{F}_{A_{n-1}}$, while the subspaces $\sigma B_{t}$ belong to $\mathcal{F}_{A_{n-1}}$. Then $\sigma \mathcal{S}=\left\{V, . ., \sigma \overline{A_{j}}, . ., \sigma B_{s}, ..\right\}$. As one can quickly check, $\sigma \mathcal{S} \in \mathcal{B}(n-1)$.

Remark 4.1. This action can also be lifted to the minimal spherical model of type $A_{n-1}$ (see for instance the exposition in [1, Section 3]).

\section{The Extended Action on Bigger Models: the Example of $A_{3}$}

From now on the minimal and the maximal models associated with the root system $A_{n-1}$ will play a special role in this paper. Hence it is convenient to single out them by a new notation.

Definition 5.1. We will denote by $Y_{\min A_{n-1}}$ the minimal model $Y_{\mathcal{F}_{A_{n-1}}}$ and by $Y_{\text {max } A_{n-1}}$ the maximal model $Y_{\mathcal{C}_{A_{n-1}}}$.

It is known that is not possible to extend the $S_{n+1}$ action from the strata of the boundary of $Y_{\min A_{n-1}}$ to the strata of the boundary of the non minimal models (see for instance [22, Remark 5.4]).

Now we want to construct a model which is 'bigger' than $Y_{\max A_{n-1}}$ (i.e. it admits a birational projection onto $\left.Y_{\max A_{n-1}}\right)$ and is equipped with an $S_{n+1}$ action. We will call supermaximal a model which is minimal among the models that have these properties. Let us construct this by an example in the case $Y_{\max A_{3}}$.

Example 5.1. We consider the action of the group $S_{5}$ on the model $Y_{\text {minA }}$ : the transposition $s_{\alpha_{0}}$ maps the 1-dimensional strata $\left\{V,<\alpha_{1}>\right\}$ and $\left\{V,<\alpha_{3}>\right\}$ as follows:

$$
s_{\alpha_{0}}\left\{V,<\alpha_{1}>\right\}=\left\{V, s_{\alpha_{0}} \overline{<\alpha_{1}>}\right\}=\left\{V,<\alpha_{1}+\alpha_{2}, \alpha_{3}>\right\}
$$

and

$$
s_{\alpha_{0}}\left\{V,<\alpha_{3}>\right\}=\left\{V, s_{\alpha_{0}}<\alpha_{3}>\right\}=\left\{V,<\alpha_{3}>\right\} .
$$

As one of the steps in the construction of $Y_{\max A_{3}}$, we blow up $Y_{\min A_{3}}$ along the intersection

$$
\left\{V,<\alpha_{1}>\right\} \cap\left\{V,<\alpha_{3}>\right\}=\left\{V,<\alpha_{1}>,<\alpha_{3}>\right\} .
$$

Hence, in order to have a model with an extended $S_{5}$ action, we also need to blow up $Y_{\max A_{3}}$ along the intersection

$$
\left\{V,<\alpha_{1}+\alpha_{2}, \alpha_{3}>\right\} \cap\left\{V,<\alpha_{3}>\right\}=\left\{V,<\alpha_{1}+\alpha_{2}, \alpha_{3}>,<\alpha_{3}>\right\} .
$$

Actually, because of the $S_{4}$ symmetry, one has to blow up in $Y_{\max A_{3}}$ all the points $\{V,\langle\alpha, \beta>,\langle\alpha\rangle\}$, where the two roots $\alpha, \beta$ span an irreducible root subsystem.

Let us denote by $Y_{\text {supermax }} A_{3}$ the model obtained as the result of all these blowups: one can immediately check that $Y_{\text {supermax } A_{3}}$ coincides with $Y_{\mathcal{B}(3)}$ and therefore the $S_{5}$ action on $Y_{\text {min } A_{3}}$ described in Section 4 extends to $Y_{\text {supermax }} A_{3}$. In the next two sections we will prove that $Y_{\mathcal{B}(n-1)}$ is a supermaximal model for every $n \geq 3$.

6. The $S_{n+1}$ ACtion on $Y_{\mathcal{B}(n-1)}$ And the minimality PROPERTy of $\mathcal{B}(n-1)$

As explained in Section 3 the strata of $Y_{\mathcal{B}(n-1)}$ are in correspondence with the elements of $\mathcal{N}^{\prime}(\mathcal{B}(n-1))$. The $S_{n+1}$ action on the open part of $Y_{\mathcal{B}(n-1)}$ can be extended to the boundary. In fact one can immediately check that the geometric $S_{n+1}$ action on $\mathcal{B}(n-1)$ can be extended to $\mathcal{N}^{\prime}(\mathcal{B}(n-1))$ : let $\left\{\{V\}, \mathcal{T}_{1}, \ldots, \mathcal{T}_{r}\right\}$ be and element of $\mathcal{N}^{\prime}(\mathcal{B}(n-1))$ and let $\sigma \in S_{n+1}$, then $\sigma$ sends $\left\{\{V\}, \mathcal{T}_{1}, \ldots, \mathcal{T}_{r}\right\}$ to $\left\{\{V\}, \mathcal{T}_{1}^{\prime}, \ldots, \mathcal{T}_{r}^{\prime}\right\}$ where, for every $i, \mathcal{T}_{i}^{\prime}=\sigma \mathcal{T}_{i}$ according to the action on $\mathcal{B}(n-1)$ 
illustrated in the end of Section 4 From the inclusions $\{V\} \subsetneq \mathcal{T}_{1} \subsetneq \cdots \subsetneq \mathcal{T}_{r}$ it immediately follows that $\{V\} \subsetneq \mathcal{T}_{1}^{\prime} \subsetneq \cdots \subsetneq \mathcal{T}_{r}^{\prime}$, therefore $\left\{\{V\}, \mathcal{T}_{1}^{\prime}, \ldots, \mathcal{T}_{r}^{\prime}\right\}$ belongs to $\mathcal{N}^{\prime}(\mathcal{B}(n-1))$.

Now we address the following combinatorial problem: what is the minimal building set in $\mathcal{B}(n-1)$ that is closed under the $S_{n+1}$ action and 'contains' $\mathcal{C}_{A_{n-1}}$ ? We start by expressing in a precise way what we mean with 'contains' $\mathcal{C}_{A_{n-1}}$.

Recall that we write $\mathcal{N}^{\prime}\left(\mathcal{C}_{A_{n-1}}\right)$ for the poset given by the nested sets in $\mathcal{C}_{A_{n-1}}$ that contain $\{V\}$, i.e. the poset that indicizes the strata of $Y_{\max A_{n-1}}$.

Proposition 6.1. There is a graded poset embedding $\varphi$ of $\mathcal{N}^{\prime}\left(\mathcal{C}_{A_{n-1}}\right)$ into $\mathcal{N}^{\prime}(\mathcal{B}(n-$ 1)).

Proof. Let $\mathcal{T}$ be an element in $\mathcal{N}^{\prime}\left(\mathcal{C}_{A_{n-1}}\right)$. Then $\mathcal{T}=\left\{B_{0}=V, B_{1}, B_{2}, \ldots, B_{r}\right\}$ is a nested set of the building set $\mathcal{C}_{A_{n-1}}$ containing $V$. This means that its elements are linearly ordered by inclusion: $V \supset B_{1} \supset \cdots \supset B_{r}$. Now we can express every $B_{i}$ as the direct sum of some irreducible subspaces $A_{i j}$, i.e. elements of $\mathcal{F}_{A_{n-1}}$ $\left(j=1, \ldots, k_{i}\right)$. We notice that, for every $i=1, \ldots, r$, the sets $\mathcal{T}_{i}^{\prime}=\left\{A_{s j}\right\} \cup\{V\}$ (with $s>r-i$ and, for every $s, j=1, \ldots, k_{s}$ ) is nested in $\mathcal{F}_{A_{n-1}}$. The map $\varphi$ defined by

if $r \geq 1$, otherwise

$$
\varphi(\mathcal{T})=\left\{\{V\}, \mathcal{T}_{1}^{\prime}, \ldots, \mathcal{T}_{r}^{\prime}\right\}
$$

is easily seen to be a poset embedding.

$$
\varphi(\mathcal{T})=\{\{V\}\}
$$

Given a complex of nested sets $P$, we will denote by $F^{k}(P)$ the subset made by the nested sets of cardinality $k+1$.

The restriction of $\varphi$ to $F^{1}\left(\mathcal{N}^{\prime}\left(\mathcal{C}_{A_{n-1}}\right)\right)$ is an embedding of $F^{1}\left(\mathcal{N}^{\prime}\left(\mathcal{C}_{A_{n-1}}\right)\right)$ into $F^{1}\left(\mathcal{N}^{\prime}(\mathcal{B}(n-1))\right)$. Now $F^{1}\left(\mathcal{N}^{\prime}(\mathcal{B}(n-1))\right)$ can be identified with $\mathcal{B}(n-1)$ (the identification maps $\{\{V\}, \mathcal{S}\}$, with $\mathcal{S}$ a nested set of $\mathcal{F}_{A_{n-1}}$ that strictly contains $V$, to $\mathcal{S})$ and we still call $\varphi$ the embedding from $F^{1}\left(\mathcal{N}^{\prime}\left(\mathcal{C}_{A_{n-1}}\right)\right)$ to $\mathcal{B}(n-1)$. More explicitly, if $B_{1} \in \mathcal{C}_{A_{n-1}}$ is a subspace which is the direct sum of the irreducible subspaces $A_{11}, \ldots, A_{1 k_{1}}$ then

$$
\varphi\left(\left\{V, B_{1}\right\}\right)=\left\{V, A_{11}, \ldots, A_{1 k_{1}}\right\} .
$$

Theorem 6.1. The minimal building subset of $\mathcal{B}(n-1)$ which contains the image $\varphi\left(F^{1}\left(\mathcal{N}^{\prime}\left(\mathcal{C}_{A_{n-1}}\right)\right)\right)$ and is closed under the $S_{n+1}$ action is $\mathcal{B}(n-1)$ itself.

Proof. Let us consider a building subset $\Gamma$ of $\mathcal{B}(n-1)$ that contains $\varphi\left(F^{1}\left(\mathcal{N}^{\prime}\left(\mathcal{C}_{A_{n-1}}\right)\right)\right)$ and is closed under the $S_{n+1}$ action. We will prove the claim by showing that $\Gamma=\mathcal{B}(n-1)$.

This can be done by induction on the depth of an element of $\mathcal{B}(n-1)$, which is defined in the following way: let $\mathcal{T}$ be a $\mathcal{F}_{A_{n-1}}$-nested set that contains $V$ and consider the levelled graph associated to $\mathcal{T}$. This graps is an oriented tree: it coincides with the Hasse diagram of the poset induced by the inclusion relation, where the leaves are the minimal subspaces of $\mathcal{S}$ and the root is $V$ and the orientation goes from the root to the leaves. A vertex $v$ is in level $k$ if the maximal length of a path that connects $v$ to a leaf is $k$. We say that $\mathcal{T}$ has depth $k$ if $k$ is the highest level of this tree. 2

\footnotetext{
${ }^{2}$ We notice that this representation of a nested set by a tree is coherent with the one introduced in the Section 2.2 the only difference is that there we added $n$ leaves.
} 
Now we prove by induction on $k$ that every element in $\mathcal{B}(n-1)$ with depth $k$ belongs to $\Gamma$.

When $k=0,1$ this is immediate: given $B \neq V \in \mathcal{C}_{A_{n-1}}$, then $\varphi(\{V, B\})$ is the nested set of depth 1 whose elements are $V$ and the maximal elements of $\mathcal{F}_{A_{n-1}}$ contained in $B$. In this way one can show that all the elements of $\mathcal{B}(n-1)$ with depth 1 that contain $V$ belong to $\Gamma$.

Let us check the case $k=2$. One first observes that, in view of the definition of the $S_{n+1}$ action, every nested set of depth 2 of the form $\left\{V, B, B_{1}\right\}$, where $B_{1} \subset B$, belongs to $\Gamma$ since it can be obtained as $\sigma \mathcal{S}$ for a suitable choice of $\sigma \in S_{n+1}$ and of a the nested set of depth $1 \mathcal{S} \in \mathcal{B}(n-1)$. Now we show that also all the nested sets of depth 2 of the form $\left\{V, B, B_{1}, \ldots, B_{j}\right\}$, with $j \geq 2$ and $B_{i} \subset B$ for every $i$, belong to $\Gamma$. In fact we can obtain $\left\{V, B, B_{1}, \ldots, B_{j}\right\}$ as a union, for every $i$, of the nested sets $\left\{V, B, B_{i}\right\}$ that belong to $\Gamma$ as remarked above. Since all these sets have a nontrivial intersection $\{V, B\}$ and $\Gamma$ is building in the Feichtner-Kozlov sense (see Section 30, this shows that $\left\{V, B, B_{1}, \ldots, B_{j}\right\}$ belongs to $\Gamma$.

Then let us consider a nested set of depth $2\left\{V, B, B_{1}, \ldots, B_{j}\right\}(j \geq 2)$, where

i) $B$ is in level 1 ;

ii) $B_{j}$ is not included in $B$;

iii) all the $B_{i}$ 's are in level 0 .

This nested set is in $\Gamma$ since it can be obtained as a union of the nested sets (with depth 1) $\mathcal{S}_{1}=\left\{V, B_{1}, \ldots, B_{j}\right\}$ and $\mathcal{S}_{2}$, where $\mathcal{S}_{2}$ is any nested subset of $\left\{V, B, B_{1}, \ldots, B_{j}\right\}$ with depth 2 . We notice that $\mathcal{S}_{1}$ and $\mathcal{S}_{2}$ are in $\Gamma$, and have nonempty intersection, therefore their union belongs to $\Gamma$.

Now we can show that in $\Gamma$ there are all the nested sets of depth 2: if the set $\left\{V, C_{1}, \ldots C_{s}, B_{1}, \ldots, B_{j}\right\}$ has depth 2 , where $s \geq 2$ and the subspaces $C_{1}, \ldots, C_{s}$ are in level 1 , while the $B_{i}$ 's are in level 0 , we can obtain $\left\{V, C_{1}, \ldots C_{s}, B_{1}, \ldots, B_{j}\right\}$ as the union of the nested sets $\left\{V, C_{i}, B_{1}, \ldots, B_{j}\right\}$ (for every $i=1, . ., s$ ) that have pairwise nonempty intersection and belong to $\Gamma$, as we have already shown.

Let us now consider $k \geq 2$ and suppose that every nested set in $\mathcal{B}(n-1)$ with depth $\leq k$ belongs to $\Gamma$. Let $\mathcal{T}$ be a nested set of depth $k+1$. Let us denote by $\mathcal{T}_{k}$ the nested set obtained removing from $\mathcal{T}$ the subspaces in level 0 : it belongs to $\Gamma$ by the inductive hypothesis. Then we consider the nested set $\mathcal{T}^{\prime}$ obtained removing from $\mathcal{T}$ the levels $2, \ldots, k$ : since $\mathcal{T}^{\prime}$ has depth 2 it belongs to $\Gamma$ again by the inductive hypothesis. We observe that $\mathcal{T}_{k}$ and $\mathcal{T}^{\prime}$ have nonempty intersection, therefore their union $\mathcal{T}$ belongs to $\Gamma$.

\section{Supermaximal MOdels AND COHOMOLOGY}

7.1. The Model $Y_{\mathcal{B}(n-1)}$ is a Supermaximal Model. We can now answer to the question, raised in Section 5, about how to construct a model that is 'bigger' than the maximal model, admits the extended $S_{n+1}$ action and is minimal with these properties.

Let us state this in a more formal way. Let us consider the poset $\mathcal{B}(n-1)$ that indicizes the strata of the minimal model $Y_{\min A_{n-1}}$, and let us denote by $\mathcal{L}$ the family of the models obtained by blowing up all the building subsets of these strata. We observe that $\mathcal{L}$ has a natural poset structure given by the relation $Y_{\mathcal{G}_{1}} \leq Y_{\mathcal{G}_{2}}$ if and only if $\mathcal{G}_{1} \subseteq \mathcal{G}_{2}$ (by Li's definition, this also means that there is a birational projection of $Y_{\mathcal{G}_{2}}$ onto $\left.Y_{\mathcal{G}_{1}}\right)$. 
Let us denote by $\mathcal{T}$ the set the elements of $\mathcal{B}(n-1)$ with depth 1 . From Theorem 2.3 and Remark 2.2 it follows that if we blowup in $Y_{\min A_{n-1}}$ the strata that correspond to the elements of $\mathcal{T}$ (in a suitable order, i.e. first the strata with smaller dimension) we obtain the model $Y_{\max A_{n-1}}$.

Definition 7.1. The supermaximal model $Y_{\text {supermax } A_{n-1}}$ associated with the root arrangement $A_{n-1}$ is the minimal model $Y_{\mathcal{K}}$ in the poset $\mathcal{L}$ that admits the $S_{n+1}$ action and such that $\mathcal{K} \supseteq \mathcal{T}$.

We notice that this last property means that the supermaximal $Y_{\text {supermax }} A_{n-1}$ model admits a birational projection onto $Y_{\max A_{n-1}}$.

As a consequence of Theorem 6.1 we have proven the following result:

Theorem 7.1. The model $Y_{\mathcal{B}(n-1)}$ is the supermaximal model associated with the root arrangement $A_{n-1}$.

Remark 7.1. There is a family of $S_{n}$-invariant building sets that are intermediate between $\mathcal{F}_{A_{n-1}}$ and $\mathcal{C}_{A_{n-1}}$ (these building sets have been classified in 22]). Let $\mathcal{E}$ be such a building set and let $Y_{\mathcal{E}}$ be the corresponding model. We will denote by $Y_{\text {superE }}$ the minimal model in $\mathcal{L}$ among the models $Y_{\mathcal{K}}$ that admit the $S_{n+1}$-action and such that $\mathcal{K} \supseteq \mathcal{E}$. Depending on the choice of $\mathcal{E}$, it may be $Y_{\text {superE }} \lessgtr Y_{\mathcal{B}(n-1)}$. This happens for instance when $\mathcal{E}$ is the building set that contains $\mathcal{F}_{n-1}$ and all the triples $\left\{V, A_{1}, A_{2}\right\} \in \mathcal{B}(n-1)$ such that the sum of $A_{1}$ and $A_{2}$ is direct and has dimension $n-2$ (this building set is denoted by $\mathcal{G}_{2}\left(A_{n-1}\right)$ in [22]).

7.2. The Cohomology of a Complex Supermaximal Model. The discussion in the preceding sections points out the interest of the supermaximal models and of the corresponding symmetric group actions.

Let $\mathcal{F}$ be the building set of irreducible subspaces associated with a subspace arrangement in a complex vector space $V$ of dimension $n$. In Section 3 we defined the building set $\mathcal{B}(n-1)=\mathcal{N}^{\prime}\left(\mathcal{F}_{A_{n-1}}\right)$, the building set for the supermaximal model for $A_{n-1}$. By analogy with this notation we write $\mathcal{B}(\mathcal{F})$ for the building set $\mathcal{N}^{\prime}(\mathcal{F})$, that generalizes in a way the idea of supermaximal model.

Let $Y_{\mathcal{B}(\mathcal{F})}$ be the model obtained by blowing up all the strata of the minimal model $Y_{\mathcal{F}}$. We recall from Example 2.2 that the strata of $Y_{\mathcal{B}(\mathcal{F})}$ are in bijection with the nested sets in $\mathcal{B}(\mathcal{F})$, according to the constructions given in 29,30].

Let us denote by $\pi_{\mathcal{F}}^{\mathcal{B}(\mathcal{F})}$ the projection from $Y_{\mathcal{B}(\mathcal{F})}$ onto $Y_{\mathcal{F}}$.

Theorem 7.2. A basis of the integer cohomology of the complex model $Y_{\mathcal{B}(\mathcal{F})}$ is given by the following monomials:

$$
\eta c_{\mathcal{S}_{1}}^{\delta_{1}} c_{\mathcal{S}_{2}}^{\delta_{2}} \cdots c_{\mathcal{S}_{k}}^{\delta_{k}}
$$

where

(1) $\mathcal{S}_{1} \subsetneq \mathcal{S}_{2} \subsetneq \ldots \subsetneq \mathcal{S}_{k}$ is a chain of $\mathcal{F}$-nested sets (possibly empty, i.e. $k=0$ ), with $\{V\} \subsetneq \mathcal{S}_{1}$

(2) the exponents $\delta_{i}$, for $i=1, \ldots, k$, satisfy the following inequalities: $1 \leq$ $\delta_{i} \leq\left|\mathcal{S}_{i}\right|-\left|\mathcal{S}_{i-1}\right|-1$, where we put $\mathcal{S}_{0}=\{V\} ;$

(3) $\eta$ belongs to $\left(\pi_{\mathcal{F}}^{\mathcal{B}(\mathcal{F})}\right)^{*} H^{*}\left(\mathcal{D}_{\mathcal{S}_{1}}\right)$ (if $k \geq 1$ ) or to $\left(\pi_{\mathcal{F}}^{\mathcal{B}(\mathcal{F})}\right)^{*} H^{*}\left(Y_{\mathcal{F}}\right)$ (if $\left.k=0\right)$ and is the image, via $\left(\pi_{\mathcal{F}}^{\mathcal{B}(\mathcal{F})}\right)^{*}$, of a monomial in the Yuzvinsky basis (see Section 2.3);

(4) the element $c_{\mathcal{S}_{i}}$ is the Chern class of the normal bundle of $L_{\mathcal{S}_{i}}$ (the proper transform of $\left.\mathcal{D}_{\mathcal{S}_{i}}\right)$ in $Y_{\mathcal{B}(\mathcal{F})}$. 
Proof. Let us fix some notation. We construct $Y_{\mathcal{B}(\mathcal{F})}$ starting from $Y_{\mathcal{F}}$ by choosing a sequence of blowups (we start by blowing up the 0-dimensional strata, then the 1-dimensional strata, and so on, see Remark 2.2). At a certain step of this blowup process we have blown up a set of strata indicized by a subset $\mathcal{A}$ of $\mathcal{B}(\mathcal{F})$. We then denote by $Y_{\mathcal{A}}$ the variety we have obtained. This means that $\mathcal{A}$ has the following property: there exists an integer $k$, with $2 \leq k \leq n+1$ such that $\mathcal{A}$ contains all the nested sets with cardinality $\geq k+1$ and doesn't contain any nested set with cardinality $<k$.

Remark 7.2. According to this notation, the variety $Y_{\mathcal{F}}$ can also be denoted by $Y_{\emptyset}$, i.e., it is the variety we have when no stratum has been blown up.

As a further notation, if $Y_{\mathcal{A}_{1}}$ and $Y_{\mathcal{A}_{2}}$ are two varieties obtained during the blowup process with $\mathcal{A}_{1} \subset \mathcal{A}_{2} \subset \mathcal{B}(\mathcal{F})$, we denote by $\pi_{\mathcal{A}_{1}}^{\mathcal{A}_{2}}: Y_{\mathcal{A}_{2}} \rightarrow Y_{\mathcal{A}_{1}}$ the blow up map. Let us recall the following lemma.

Lemma 7.1 (Keel, 25). Given $\mathcal{A}$ as before, let us suppose that the next stratum that we have to blow up is indicized by $\mathcal{S} \in \mathcal{B}(\mathcal{F}) \backslash \mathcal{A}$ with $|\mathcal{S}|=k$. We call $\mathcal{D}_{\mathcal{S}}^{\prime}$ the proper transform of $\mathcal{D}_{\mathcal{S}}$ in $Y_{\mathcal{A}}$ and $\widetilde{\mathcal{D}_{\mathcal{S}}}$ the proper transform of $\mathcal{D}_{\mathcal{S}}$ in $Y_{\mathcal{A} \cup\{\mathcal{S}\}}$. Then

$$
H^{*}\left(Y_{\mathcal{A} \cup\{\mathcal{S}\}}\right) \cong H^{*}\left(Y_{\mathcal{A}}\right)[\zeta] /\left(J \cdot \zeta, P_{Y_{\mathcal{A}} / \mathcal{D}_{\mathcal{S}}^{\prime}}(-\zeta)\right)
$$

where $\zeta$ is the class of the proper transform $\widetilde{\mathcal{D}_{\mathcal{S}}}$ of $\mathcal{D}_{\mathcal{S}}$ in $H^{2}\left(Y_{\mathcal{A} \cup\{\mathcal{S}\}}\right), J$ is the kernel of the restriction map $H^{*}\left(Y_{\mathcal{A}}\right) \rightarrow H^{*}\left(\mathcal{D}_{\mathcal{S}}^{\prime}\right)$ and $P_{Y_{\mathcal{A}} / \mathcal{D}_{\mathcal{S}}^{\prime}}[x]$ is any polynomial in $H^{*}\left(Y_{\mathcal{A}}\right)[x]$ whose restriction to $H^{*}\left(\mathcal{D}_{\mathcal{S}}^{\prime}\right)[x]$ is the Chern polynomial of the normal bundle of $\mathcal{D}_{\mathcal{S}}^{\prime}$ in $Y_{\mathcal{A}}$.

Using the lemma we can prove the claim of the theorem by induction. We fix a sequence of blowups

$$
X_{0} \leftarrow^{\pi} X_{1} \leftarrow^{\pi} \cdots \leftarrow X_{N}
$$

that constructs $Y_{\mathcal{B}(\mathcal{F})}=X_{N}$ starting from $Y_{\mathcal{F}}=X_{0}$. We assume by inductive hypothesis that a basis of the integer cohomology of $X_{n}$ is given by the monomials

where

$$
\eta c_{\mathcal{S}_{1}}^{\delta_{1}} c_{\mathcal{S}_{2}}^{\delta_{2}} \cdots c_{\mathcal{S}_{k}}^{\delta_{k}}
$$

(1) $\mathcal{S}_{1} \subsetneq \mathcal{S}_{2} \subsetneq \cdots \subsetneq \mathcal{S}_{k}$ is a possibly empty chain of $\mathcal{F}$-nested sets already blown up;

(2) $\eta$ belongs to $\left(\pi_{X_{0}}^{X_{n}}\right)^{*} \iota^{*} H^{*}\left(\mathcal{D}_{\mathcal{S}_{1}}\right)$ if $k \geq 1$ ( $\iota$ is the inclusion map of $\mathcal{D}_{\mathcal{S}_{i}}$ in $\left.X_{0}\right)$, and to $\left(\pi_{X_{0}}^{X_{n}}\right)^{*} H^{*}\left(X_{0}\right)$ if $k=0$ and is the image of a monomial in the Yuzvinsky basis;

(3) the term $c_{\mathcal{S}_{i}}$ is the Chern class of the normal bundle of $\mathcal{D}_{\mathcal{S}_{i}}^{\prime}$, that is the proper transform of $\mathcal{D}_{\mathcal{S}}$ in $X_{n}$;

(4) the exponents $\delta_{i}$ satisfy the inequalities $1 \leq \delta_{i} \leq\left|\mathcal{S}_{i}\right|-\left|\mathcal{S}_{i-1}\right|-1$, where we put $\mathcal{S}_{0}=\{V\}$.

Let $\mathcal{D}_{\mathcal{S}}^{\prime}$ be the stratum in $X_{n}$ that we have to blowup in order to get $X_{n+1}$. We assume that $\mathcal{D}_{\mathcal{S}}^{\prime}$ is the proper transform in $X_{n}$ of the stratum $\mathcal{D}_{\mathcal{S}}$ in $X_{0}$, corresponding to the $\mathcal{F}$-nested set $\mathcal{S} \in \mathcal{B}(\mathcal{F})$. Let $d=|\mathcal{S}|$. Since at the step $X_{n}$ we have already blown up all the strata $\mathcal{D}_{\mathcal{T}}$ with $|\mathcal{T}|>d$, the submanifold $\mathcal{D}_{\mathcal{S}}^{\prime}$ is a complex supermaximal model of smaller dimension. In fact, since we have that, as in Definition 2.9

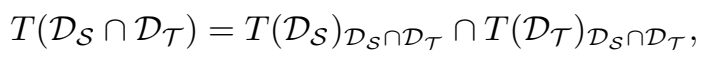


the stratum $\mathcal{D}_{\mathcal{S}}^{\prime}$ can be obtained as a blowup of $\mathcal{D}_{\mathcal{S}}$ along the strata $\mathcal{D}_{\mathcal{T}} \cap \mathcal{D}_{\mathcal{S}}$ with $|\mathcal{T}|>d$. Hence we can assume by induction on the dimension of $\mathcal{D}_{\mathcal{S}}^{\prime}$, that we know the cohomology $H^{*}\left(\mathcal{D}_{\mathcal{S}}^{\prime}\right)$, according to the statement of the theorem.

Then, by Lemma 7.1 we have that the cohomology of $X_{n+1}$ is given by

$$
H^{*}\left(X_{n+1}\right) \cong H^{*}\left(X_{n}\right)[\zeta] /\left(J \cdot \zeta, P_{X_{n} / \mathcal{D}_{\mathcal{S}}^{\prime}}(-\zeta)\right)
$$

where $J$ is the kernel of the projection induced by the inclusion of $\mathcal{D}_{\mathcal{S}}^{\prime}$ in $X_{n}$ and $\zeta=c_{\mathcal{S}}$ is the Chern class of the normal bundle of the proper transform $\widetilde{\mathcal{D}_{\mathcal{S}}}$ of $\mathcal{D}_{\mathcal{S}}$ in $X_{n+1}$. The polynomial $P_{X_{n} / \mathcal{D}_{\mathcal{S}}^{\prime}}(-\zeta)$ is the Chern polynomial of the normal bundle of $\mathcal{D}_{\mathcal{S}}^{\prime}$ in $X_{n}$, that has rank $d=|\mathcal{S}|$ and hence $\operatorname{deg} P_{X_{n} / \mathcal{D}_{\mathcal{S}}^{\prime}}(-\zeta)=d-1$.

It follows that a basis of $H^{*}\left(X_{n+1}\right)$ is given by the union of two set of generators:

(1) the monomials

$$
\mu_{1}=\eta c_{\mathcal{S}_{1}}^{\delta_{1}} c_{\mathcal{S}_{2}}^{\delta_{2}} \cdots c_{\mathcal{S}_{k}}^{\delta_{k}}
$$

that are already in the base of $H^{*}\left(X_{n}\right)$ and that we can identify with the corresponding generators of the cohomology of $X_{n+1}$ via the pull back $\pi^{*}: H^{*}\left(X_{n}\right) \rightarrow H^{*}\left(X_{n+1}\right)$

(2) the monomials

$$
\mu_{2}=\eta c_{\mathcal{S}}^{\delta} c_{\mathcal{S}_{1}}^{\delta_{1}} c_{\mathcal{S}_{2}}^{\delta_{2}} \cdots c_{\mathcal{S}_{k}}^{\delta_{k}}
$$

where $0<\delta<|\mathcal{S}|-1$ and $\eta c_{\mathcal{S}_{1}}^{\delta_{1}} c_{\mathcal{S}_{2}}^{\delta_{2}} \cdots c_{\mathcal{S}_{k}}^{\delta_{k}}$ is a monomial among the generators of the cohomology $H^{*}\left(\mathcal{D}_{\mathcal{S}}^{\prime}\right)$; we identify $\mu_{2}$ with a generator of the cohomology of $H^{*}\left(X_{n+1}\right)$ as follows: given the monomial $\eta c_{\mathcal{S}_{1}}^{\delta_{1}} c_{\mathcal{S}_{2}}^{\delta_{2}} \cdots c_{\mathcal{S}_{k}}^{\delta_{k}} \in$ $H^{*}\left(\mathcal{D}_{\mathcal{S}}^{\prime}\right)$, we identify it with the coset $\eta c_{\mathcal{S}_{1}}^{\delta_{1}} c_{\mathcal{S}_{2}}^{\delta_{2}} \cdots c_{\mathcal{S}_{k}}^{\delta_{k}}+J \in H^{*}\left(X_{n}\right)$ by the projection induced by the inclusion of $\mathcal{D}_{\mathcal{S}}^{\prime}$ in $X_{n}$; hence when we multiply it by $c_{\mathcal{S}}$, that is the Chern class of the normal bundle of the divisor $\widetilde{\mathcal{D}_{\mathcal{S}}} \in X_{n+1}$, this gives a well defined class

$$
\eta c_{\mathcal{S}}^{\delta} c_{\mathcal{S}_{1}}^{\delta_{1}} c_{\mathcal{S}_{2}}^{\delta_{2}} \cdots c_{\mathcal{S}_{k}}^{\delta_{k}} \in H^{*}\left(X_{n+1}\right) .
$$

In particular, since we are considering the class $c_{\mathcal{S}_{1}}$ that is the Chern class of the normal bundle of the proper transform $\mathcal{D}_{\mathcal{S}_{1}}^{\prime}$ of $\mathcal{D}_{\mathcal{S}_{1}}$ in $\mathcal{D}_{\mathcal{S}}^{\prime}$, the exponent $\delta_{1}$ will be at most $\left|\mathcal{S}_{1}\right|-|\mathcal{S}|-1$, that is the dimension of the projectivized normal bundle of $\mathcal{D}_{\mathcal{S}_{1}}^{\prime}$ in $\mathcal{D}_{\mathcal{S}}^{\prime}$.

Let $\mathcal{S}, \mathcal{T}$, with $\mathcal{S} \subset \mathcal{T}$ be two $\mathcal{F}$-nested sets. Let $\mathcal{D}_{\mathcal{S}}^{\prime}$ (resp. $\mathcal{D}_{\mathcal{T}}^{\prime}$ ) be the proper transform of $\mathcal{D}_{\mathcal{S}}\left(\right.$ resp. $\left.\mathcal{D}_{\mathcal{T}}\right)$ in $X_{n}$. We assume that in $X_{n}$ we have already performed the blowup of the stratum associated to $\mathcal{D}_{\mathcal{T}}$. Since we have the inclusion $\mathcal{D}_{\mathcal{T}} \subset \mathcal{D}_{\mathcal{S}}$, we can also consider the proper transform $\mathcal{D}_{\mathcal{T}}^{\prime} \cap \mathcal{D}_{\mathcal{S}}^{\prime}$ of $\mathcal{D}_{\mathcal{T}}$ in $\mathcal{D}_{\mathcal{S}}^{\prime}$.

Let $\zeta_{\mathcal{T}}$ be the Chern class of the normal bundle of $\mathcal{D}_{\mathcal{T}}^{\prime}$ in $X_{n}$ and let $\zeta_{\mathcal{T}}^{\mathcal{S}}$ be the Chern class of the normal bundle of $\mathcal{D}_{\mathcal{T}}^{\prime} \cap \mathcal{D}_{\mathcal{S}}^{\prime}$ in $\mathcal{D}_{\mathcal{S}}^{\prime}$. We claim that the projection $i^{*}$ induced by the inclusion $i: \mathcal{D}_{\mathcal{S}}^{\prime} \subset X_{n}$ maps $i^{*}: \zeta_{\mathcal{T}} \mapsto \zeta_{\mathcal{T}}$. This follows since the Thom class $\tau_{\mathcal{T}}$ of the normal bundle of $\mathcal{D}_{\mathcal{T}}^{\prime}$ in $X_{n}$ restricts to the Thom class $\tau_{\mathcal{T}}^{S}$ of the normal bundle of $\mathcal{D}_{\mathcal{T}}^{\prime} \cap \mathcal{D}_{\mathcal{S}}^{\prime}$ in $\mathcal{D}_{\mathcal{S}}$ and we have that the Chern class of a line bundle is the pullback of the zero-section of its Thom class.

Finally we notice that, since Chern classes are functorial, whenever we perform a blowup $\pi: X_{n+1} \rightarrow X_{n}$, the Chern class $c_{\mathcal{T}}$ of a divisor $\mathcal{D}_{\mathcal{T}}^{\prime}$ in $X_{n}$ pulls back to the Chern class of its proper transform $\widetilde{\mathcal{D}_{\mathcal{T}}}$ and hence we can identify them in our notation. 


\section{Poincaré SERIES AND EUler ChARACTERISTIC OF SUPERMAXIMAL MOdELS}

In this section we use the cohomology basis described in Theorem 7.2 to prove a formula for a series that encodes all the information regarding the Poincaré polynomials of the supermaximal models $Y_{\mathcal{B}(n-1)}$.

We start by recalling the analogue computation in the case of minimal models. The Poincaré series

$$
\Phi(q, t)=t+\sum_{n \geq 2} \sum_{i} \operatorname{dim} H^{2 i}\left(Y_{\min A_{n-1}}, \mathbb{Z}\right) q^{i} \frac{t^{n}}{n !}
$$

for the minimal De Concini-Procesi models $Y_{m i n A_{n-1}}$ has been computed in many different ways. One can see for instance [31], [40], 17], [23]; a formula for another series that encodes the same information is provided in Section 10 of the present paper.

Both in Section 5 of $[40$ and Section 4 of [17] the computation of $\Phi(q, t)$ consists in counting the elements of the Yuzvinski basis for $H^{*}\left(Y_{\min A_{n-1}}, \mathbb{Z}\right)$ described in Section 2.3. We recall that, given a monomial $m_{f}$ in this basis, the set supp $f$ is a nested set and that in Section 2.2 we represented the $\mathcal{F}_{A_{n-1}}$ - nested sets, i.e. the elements of $\mathcal{N}\left(\mathcal{F}_{A_{n-1}}\right)$, as oriented forests on $n$ leaves. Let us then denote by $\lambda(q, t)$ the contribution to $\Phi(q, t)$ provided by the basis monomials whose associated nested set is represented by a tree.

It turns out that $\lambda(q, t)$ satisfies the following recursive relation:

$$
\lambda(q, t)^{(1)}=1+\frac{\lambda(q, t)^{(1)}}{q-1}\left[e^{q \lambda(q, t)}-q e^{\lambda(q, t)}+q-1\right]
$$

(here the superscript ${ }^{(1)}$ means the first derivative with respect to $t$ ). Then one obtains a formula for $\Phi(q, t)$ by observing that $\Phi(q, t)=e^{\lambda(q, t)}-1$.

Let us now denote by $\Phi_{\text {super }}(q, t)$ the Poincaré series:

$$
\Phi_{\text {super }}(q, t)=t+\sum_{n \geq 2} \sum_{i} \operatorname{dim} H^{2 i}\left(Y_{\mathcal{B}(n-1)}, \mathbb{Z}\right) q^{i} \frac{t^{n}}{n !}
$$

Definition 8.1. We define the following series in four variables:

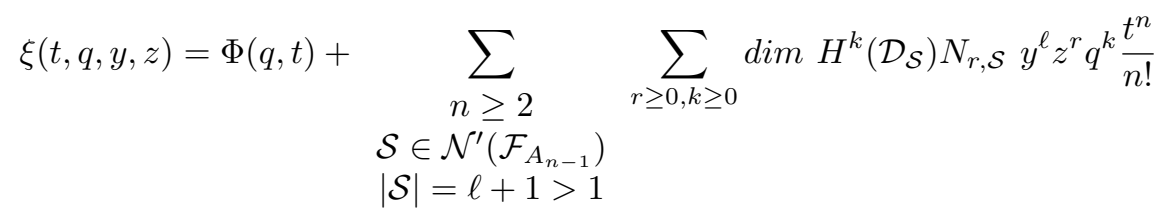

where $\mathcal{D}_{\mathcal{S}}$ is the subvariety in the boundary of $Y_{\text {min } A_{n-1}}$ defined in Section 2.3 and $N_{r, \mathcal{S}}$ is the number of nested sets in $\mathcal{N}^{\prime}\left(\mathcal{F}_{A_{n-1}}\right)$ that contain $\mathcal{S}$ and whose cardinality is $|\mathcal{S}|+r$.

Theorem 8.1. One can obtain the Poincaré series $\Phi_{\text {super }}(q, t)$ from the series $\xi(t, q, y, z)$ by substituting:

- $y^{\ell}$ with $\frac{q^{\ell}-q}{q-1}$;

- $z^{r}$ with

$$
\sum_{s \leq r, j_{0}=0<j_{1}<\cdots<j_{s}=r} \frac{r !}{j_{1} !\left(j_{2}-j_{1}\right) ! \cdots\left(j_{s}-j_{s-1}\right) !} \prod_{\theta=1}^{s} q \frac{q^{j_{\theta}-j_{\theta-1}-1}-1}{q-1}
$$


Proof. The monomials in the basis of $H^{*}\left(Y_{\mathcal{B}(n-1)}\right)(n \geq 2)$ are described by Theorem 7.2

$$
\eta c_{\mathcal{S}_{1}}^{\delta_{1}} c_{\mathcal{S}_{2}}^{\delta_{2}} \cdots c_{\mathcal{S}_{k}}^{\delta_{k}}
$$

where $\eta$ is represented by a monomial in the basis of $H^{*}\left(\mathcal{D}_{\mathcal{S}_{1}}\right)$ if $k \geq 1$ and of $H^{*}\left(Y_{\min A_{n-1}}\right)$ if $k=0$. The monomials with $k=0$ are computed by the series $\Phi(q, t)$ that is the first addendum in the formula (1). Then we observe that, once $\mathcal{S}_{1}$ is fixed, if the exponent of the variable $z$ is $r$ this means that we are keeping into account all the monomials such that $\left|\mathcal{S}_{k}-\mathcal{S}_{1}\right|=r$. This is expressed by the substitution formula for $z^{r}$.

The exponent of the variable $y$ coincides with $\left|\mathcal{S}_{1}-\{V\}\right|$ and the exponent $\delta_{1}$ satisfies $1 \leq \delta_{1} \leq\left|S_{1}-\{V\}\right|-1$ : this is expressed by the substitution of $y^{\ell}$ with $\frac{q^{\ell}-q}{q-1}$ (notice that $\left.\ell>1\right)$.

We are therefore interested in finding a formula for $\xi(t, q, y, z)$. Let us denote by $p_{n}(q)$, for $n \geq 2$, the Poincaré polynomial of the minimal model $Y_{\min A_{n-1}}$, i.e. we can write $\Phi(q, t)=t+\sum_{n \geq 2} p_{n}(q) \frac{t^{n}}{n !}$. Then we consider the series $W(t, z)=$ $\sum_{n \geq 2} w_{n}(z) t^{n}$ where the polynomials $w_{n}(z)$ count the number of elements of $\mathcal{B}(n-$ 1). More precisely

$$
w_{n}(z)=\sum_{1 \leq j \leq n-1} \sum_{\substack{\mathcal{S} \in \mathcal{B}(n-1) \\|\mathcal{S}|=j}} z^{j-1}
$$

As we will recall in Section 9, we have

$$
w_{n}(z)=\sum_{1 \leq j \leq n-1}\left|\mathcal{P}_{2}(n+j-1, j)\right| z^{j-1}
$$

where we denote by $\mathcal{P}_{2}(n+j-1, j)$ the number of unordered partitions of the set $\{1, \ldots, n+j-1\}$ into $j$ parts of cardinality greater than or equal to 2 (these numbers are the 2-associated Stirling numbers of the second kind, see for instance the table at page 222 of $[3]$ ).

If we now put

$$
\psi(t, q, z)=\sum_{n \geq 2} p_{n}(q) w_{n}(z) \frac{t^{n}}{n !}
$$

we can write a formula that computes the series $\xi(t, q, y, z)$ :

Theorem 8.2. We have

$$
\xi(t, q, y, z)=\Phi(q, t)+\sum_{\nu \geq 1} \psi(t, q, z)^{(\nu)} \frac{\Gamma^{\nu}}{\nu !}
$$

where

$$
\Gamma=\sum_{\ell \geq 1} \frac{1}{\ell !} y^{\ell}\left(\psi(t, q, z)^{\ell}\right)^{(\ell-1)}
$$

and the superscript ${ }^{(j)}$ means the $j$-th derivative with respect to $t$.

The next (sub)section will be devoted to the proof of this theorem. 
Example 8.1. Here it is a computation of the first terms of the series $\xi(t, q, y, z)$, that is the terms whose $t$-degree is $\leq 5$ :

$$
\begin{aligned}
& \frac{t^{5}}{5 !}\left[\left(315 q^{2}+1305 q+315\right) y z^{2}+\left((315 q+315) y^{2}+\left(210 q^{2}+870 q+210\right) y\right) z+\right. \\
& \left.\quad+105 y^{3}+(105 q+105) y^{2}+\left(25 q^{2}+95 q+25\right) y+q^{3}+16 q^{2}+16 q+1\right]+ \\
& +\frac{t^{4}}{4 !}\left[30 y z(1+q)+15 y^{2}+10 y(1+q)+q^{2}+5 q+1\right]+\frac{t^{3}}{3 !}[3 y+q+1]+\frac{t^{2}}{2}+t .
\end{aligned}
$$

If we put $q=1$ in the formula for the Poicaré series $\Phi_{\text {super }}(q, t)$, we obtain the Euler characteristic series of the supermaximal models $Y_{\mathcal{B}(n-1)}$. Moreover we notice that if we put $q=-1$ we obtain the Euler characteristic series of the real supermaximal models $Y_{\mathcal{B}(n-1)}(\mathbb{R})$ constructed with base field $\mathbb{R}$. In fact from a result of 26 it follows that $H^{2 i}\left(Y_{\mathcal{B}(n-1)}, \mathbb{Z}_{2}\right) \cong H^{i}\left(Y_{\mathcal{B}(n-1)}(\mathbb{R}), \mathbb{Z}_{2}\right)$; therefore $\sum_{i}(-1)^{i} \operatorname{dim} H^{2 i}\left(Y_{\mathcal{B}(n-1)}, \mathbb{Q}\right)$ is equal to the Euler characteristic $\chi_{E}\left(Y_{\mathcal{B}(n-1)}(\mathbb{R})\right)$.

We observe that one can obtain the Euler characteristic series in a more direct way from the series $\xi(t, q, y, z)$ :

Theorem 8.3. One can compute the Euler characteristic series of the models $Y_{\mathcal{B}(n-1)}(\mathbb{R})$ from the series $\xi(t, q, y, z)$ by substituting $q$ with -1 and also

- $y^{k}$ with -1 if $k$ is even, otherwise with 0 ;

- $z^{r}$ with $E_{r}$, where we denote by $E_{n}$ the Euler secant number defined by

$$
\frac{2}{e^{t}+e^{-t}}=\sum_{n} E_{n} \frac{t^{n}}{n !}
$$

(see sequence A028296 in OEIS; notice that if $n$ is odd then $E_{n}=0$ ).

Proof. When we find $z^{r}$ in $\xi(t, q, y, z)$, it means that we are computing the contribution to the Euler characteristic of all the factors $c_{\mathcal{S}_{1}}^{\delta_{1}} c_{\mathcal{S}_{2}}^{\delta_{2}} \cdots c_{\mathcal{S}_{k}}^{\delta_{k}}$ of the basis elements, such that $\left|\mathcal{S}_{k}-\mathcal{S}_{1}\right|=r$. We observe that this contribution is non zero if and only if $r$ is even. In this case our problem is equivalent to computing the Euler characteristic of the order complex of the poset $\mathcal{P}(\{1, \ldots, r\})_{\text {even }}$ of the subsets of $\{1, \ldots, r\}$ with even cardinality. By Philip Hall Theorem (see for instance Proposition 1.2.6 of [39]) we can compute it via the Moebius function, that is equal to $E_{r}$ (see Section 3.7 of Stanley's survey [37).

8.1. Proof of Theorem $\mathbf{8 . 2}$. We will follow a strategy similar to the one in Section 3 of [18].

Definition 8.2. Given any rooted oriented tree $T$, the polynomial $\mathcal{Q}(T)$ is a product of monomials that contains a factor for each vertex of $T$ : if $v$ is a vertex of $T$ with $\nu$ outgoing edges, the corresponding factor is $y^{\nu} \psi(t, q, z)^{(\nu)}$.

We notice that the cardinality $A u t(T)$ of the automorphism group of a rooted tree $T$ is equal to the product $\prod_{v} \gamma_{s y m, v}$ where $v$ ranges over the vertices of $T$ and $\gamma_{s y m, v}$ is determined in this way: delete $v$ and consider the connected components of the subgraph of $T$ that stems from $v$. Suppose that they can be partitioned in $k$ automorphism classes with the following cardinalities: $a_{1}, a_{2}, \ldots, a_{k}$. Then $\gamma_{s y m, v}=a_{1} ! a_{2} ! \cdots a_{k} !$ 
Now we associate a tree to every monomial $\eta c_{\mathcal{S}_{1}}^{\delta_{1}} c_{\mathcal{S}_{2}}^{\delta_{2}} \cdots c_{\mathcal{S}_{k}}^{\delta_{k}}$ in the basis of $H^{*}\left(Y_{\mathcal{B}(n-1)}\right)$, by choosing the rooted tree with $n$ leaves associated with the nested set $\mathcal{S}_{1}$ (see Section 2.2 and deleting the leaves and the edges that contain a leaf. Then $\xi(t, q, y, z)-\Phi(q, t)$ can be computed by regrouping together all the basis monomials that are associated with the same rooted tree. This means that $\xi(t, q, y, z)-\Phi(q, t)$ can be written as:

$$
\sum_{n \geq 1} \psi(t, q, z)^{(n)} \frac{(y \Gamma)^{n}}{n !}
$$

where

$$
\Gamma=\sum_{[T]} \frac{\mathcal{Q}(T)}{A u t(T)}
$$

and the sum ranges over all the automorphism classes of nonempty oriented, rooted trees.

Thus the problem can be reduced to the one of finding a 'nice' formula for $\Gamma$. This is provided by the following theorem, that is a consequence of Theorem 3.3 of [18].

Theorem 8.4.

$$
\sum_{[T]} \frac{\mathcal{Q}(T)}{\operatorname{Aut}(T)}=\sum_{n \geq 1} \frac{1}{n !} y^{n-1}\left(\psi(t, q, z)^{n}\right)^{(n-1)}
$$

where $[T]$ ranges over all the automorphism classes of nonempty oriented rooted trees.

\section{A combinatorial extended $S_{n+k}$ ACtion on the poset of the BOUNDARY STRATA OF $Y_{\mathcal{F}_{A_{n-1}}}$}

This section and the next one are devoted to point out that another hidden extended action of the symmetric group appears in the geometry of the minimal models $Y_{\mathcal{F}_{A_{n-1}}}$. This action is different from the one, described in Section 4, that motivated the construction of supemaximal models. More precisely, we are going to deal with a purely combinatorial action on the poset $\mathcal{B}(n-1)$ that indexes the strata of $Y_{\mathcal{F}_{A_{n-1}}}$. This does not correspond to an action on the variety $Y_{\mathcal{F}_{A_{n-1}}}$, but it gives rise, as we will see, to an interesting permutation action on the monomials of the Yuzvinski basis of $H^{*}\left(Y_{\mathcal{F}_{A_{n-1}}}, \mathbb{Z}\right)$.

Let us denote by $F^{k}(\mathcal{B}(n-1))$ the subset of $\mathcal{B}(n-1)$ made by the elements of cardinality $k+1$. These elements indicize the $k$-codimensional strata of $Y_{\mathcal{F}_{A_{n-1}}}$ (the only element in $F^{0}(\mathcal{B}(n-1))$ is $\{V\}$ that corresponds to the big open part). In 20 it has been described an explicit bijection between $F^{k}(\mathcal{B}(n-1))$ and the set of unordered partitions of $\{1,2, \ldots, n+k\}$ into $k+1$ parts of cardinality greater than or equal to 2 . To recall this bijection, we identify the elements of $\mathcal{F}_{A_{n-1}}$ with subsets of $\{1,2, \ldots n\}$, as in Section 2.2 .

Definition 9.1. We fix the following (strict) partial ordering on $\mathcal{F}_{A_{n-1}}$ : given I and $J$ in $\mathcal{F}_{A_{n-1}}$ we put $I<J$ if the minimal number in $I$ is less than the minimal number in $J$.

Let us consider a nested set $\mathcal{S}$ that belongs to $F^{k}(\mathcal{B}(n-1))$. It can be represented by an oriented rooted tree on $n$ leaves as in Section 2.2. The leaves are the sets 
$\{1\},\{2\}, \cdots,\{n\}$. Now we put labels on the vertices of this tree. We start by labelling the vertices $\{1\},\{2\}, \cdots,\{n\}$ respectively by the labels $1,2, \ldots, n$.

Then we can partition the set of vertices of the tree into levels with the same criterion as in the proof of Theorem6.1 level 0 is made by the leaves, and in general, level $j$ is made by the vertices $v$ such that the maximal length of an oriented path that connects $v$ to a leaf is $j$.

Now we label the internal vertices of the tree in the following way. Let us suppose that there are $q$ vertices in level 1 . These vertices correspond, by the nested property, to pairwise disjoint elements of $\mathcal{F}_{A_{n-1}}$, therefore we can totally order them using the ordering of Definition 9.1 and we label them with the numbers from $n+1$ to $n+q$ (the label $n+1$ goes to the minimum, while $n+q$ goes to the maximum).

At the same way, if there are $t$ vertices in level 2, we can label them with the numbers from $n+q+1$ to $n+q+t$, and so on. At the end of the process, the root is labelled with the number $n+k+1$.

We can now associate to such a tree an unordered partition of $\{1,2, \ldots, n+k\}$ into $k+1$ parts by assigning to every internal vertex $v$ the set of the labels of the vertices covered by $v$ (see Figure 1).

$$
\begin{aligned}
S=\{ & \{2,3,4\},\{1,6\},\{5,7\},\{2,3,4,8\}, \\
& \{1,5,6,7\},\{1,2,3,4,5,6,7,8,9\}\}
\end{aligned}
$$

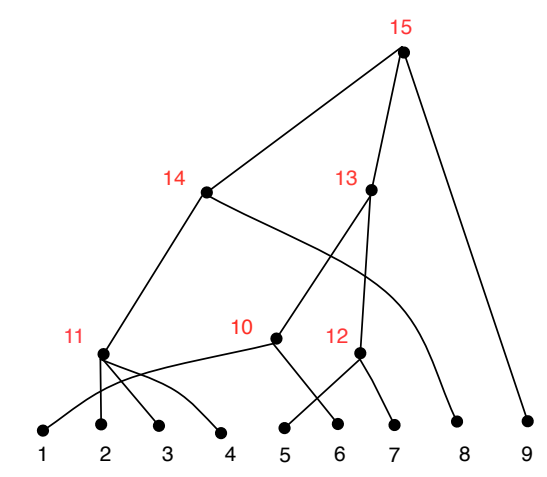

$\{1,6\}\{2,3,4\}\{5,7\}\{8,11\}\{10,12\}\{9,13,14\}$

Figure 1. On top of the picture there is a nested set $S$ with 6 elements in $\mathcal{F}_{A_{8}}$. In the middle there is its representation by an oriented labelled rooted tree. At the bottom one can read the resulting partition of $\{1,2, \ldots, 14\}$ into 6 parts.

This bijection allows us to consider new actions of the symmetric group on $\mathcal{B}(n-1)$ : every subset $F^{k}(\mathcal{B}(n-1))$ is equipped with an action of $S_{n+k}$.

Remark 9.1. We notice that when $k>2$, if we first embed $S_{n}$ into $S_{n+k}$ in the standard way and then restrict the $S_{n+k}$ action to $S_{n}$ we do not obtain the natural $S_{n}$ action on $\mathcal{B}(n-1)$. For instance, let us consider $n=5$ and $k=3$, and the 
following nested set $\mathcal{S}$ in $\mathcal{B}(4)$ :

$$
\mathcal{S}=\{\{1,2\},\{3,4\},\{3,4,5\},\{1,2,3,4,5\}\}
$$

On one hand, the natural action of the transposition $(1,3)$ sends $\mathcal{S}$ to

$$
\mathcal{S}^{\prime}=\{\{2,3\},\{1,4\},\{1,4,5\},\{1,2,3,4,5\}\}
$$

On the other hand, the partition of $\{1,2,3, . ., 8\}$ associated with $\mathcal{S}$ is

$$
\{1,2\},\{3,4\},\{5,7\},\{6,8\}
$$

that is sent by the transposition $(1,3)$ to $\{2,3\},\{1,4\},\{5,7\},\{6,8\}$.

This last partition corresponds to the nested set

$$
\mathcal{S}^{\prime \prime}=\{\{1,4\},\{2,3\},\{2,3,5\},\{1,2,3,4,5\}\}
$$

and we notice that $\mathcal{S}^{\prime} \neq \mathcal{S}^{\prime \prime}$.

Moreover, we observe that the natural $S_{5}$ action on $F^{3}(\mathcal{B}(4))$ and the $S_{5}$ action restricted from $S_{8}$ differ in the number of orbits, therefore when we consider the associated permutation representations they differ in the multiplicity of the trivial representation, which is 3 for the natural $S_{5}$ representation and 4 for the restricted one.

\section{The $S_{n+k}$ ACTION ON THE BASIS OF $H^{*}\left(Y_{\mathcal{F}_{A_{n-1}}}, \mathbb{Z}\right)$}

As we observed in the preceding section, the combinatorial action of $S_{n+k}$ on $F^{k}(\mathcal{B}(n-1))$ can be read as an action on the $k$-codimensional strata of $Y_{\mathcal{F}_{A_{n-1}}}$. Moreover we notice that this action can in turn be extended to the Yuzvinski basis of $H^{*}\left(Y_{\mathcal{F}_{A_{n-1}}}, \mathbb{Z}\right)$ described in Section 2.3. In fact we can represent the elements of the Yuzvinski basis by labelled partitions in the way illustrated by the following example.

Example 10.1. Let $n=7$ and let us consider the monomial $c_{A_{1}}^{2} c_{A_{2}}$ in the Yuzvinski basis of $H^{6}\left(Y_{\mathcal{F}_{A_{6}}}, \mathbb{Z}\right)$, where $\left\{A_{1}, A_{2}\right\}$ is the nested set given by the subspaces $A_{1}=\{1,2,3,5\}, A_{2}=\{4,6,7\}$. Since $V$ does not belong to this nested set, we write this monomial as $c_{A_{1}}^{2} c_{A_{2}} c_{V}^{0}$. Now, according to the bijection described in Section 9. we can associate to the nested set $\left\{V, A_{1}, A_{2}\right\}$ the following partition of the set $\{1,2, . ., 9\}$ :

$$
\{1,2,3,5\}\{4,6,7\}\{8,9\}
$$

where, $A_{1}$ corresponds to $\{1,2,3,5\}, A_{2}$ corresponds to $\{4,6,7\}$ and $V$ corresponds to $\{8,9\}$. Finally we associate to $c_{A_{1}}^{2} c_{A_{2}}$ the following labelled partition of $\{1,2, . ., 9\}$ :

$$
\{1,2,3,5\}^{2}\{4,6,7\}^{1}\{8,9\}^{0}
$$

As another example, we represent the monomial $c_{A_{1}}^{2} c_{V}^{2}$ of $H^{8}\left(Y_{\mathcal{F}_{A_{6}}}, \mathbb{Z}\right)$ by the labelled partition of $\{1,2, . ., 8\}$ :

$$
\{1,2,3,5\}^{2}\{4,6,7,8\}^{2}
$$

We notice that this representation provides us with an easy way to 'read' the bounds for the exponents in the Yuzvinski basis (see the end of Section 2.3). More in detail, the bounds $d_{(\operatorname{supp} f)_{A}, A}^{\{V\}}$ can be translated in this language in the following way. Let $I$ be a part of a labelled partition of $\{1, \ldots, n+k\}$ that represents a monomial in the Yuzvinski basis: then the exponent (i.e. the label) $\alpha_{I}$ of $I$ satisfies $0 \leq \alpha_{I} \leq|I|-2$. Moreover, it may be equal to 0 only if $I$ contains the number $n+k$, 
i.e. when $I$ represents $V$, and in the monomial the variable $c_{V}$ does not appear (that is, according to the convention established before, it appears with exponent $0)$. In particular all the sets in the partition have cardinality $\geq 3$ except possibly for the set containing $n+k$, that may have cardinality equal to 2 .

Now we observe that $S_{n+k}$ acts on the labelled partitions of $\{1, \ldots, n+k\}$ into $k+1$ parts, and this provides us with a permutation action on the monomials of the Yuzvinski basis of $H^{*}\left(Y_{\mathcal{F}_{A_{n-1}}}, \mathbb{Z}\right)$. More in detail:

- $S_{n+k}$ acts on the set of all the monomials that are represented by a labelled partition of $\{1, \ldots, n+k\}$ into $k+1$ parts with all the labels $>0$.

- $S_{n+k-1}$ acts on the set of all the monomials that are represented by a labelled partition of $\{1, \ldots, n+k\}$ into $k+1$ parts with one of the labels equal to 0 . In fact if there is a part labelled by 0 , it must contain the number $n+k$, and $S_{n+k-1}$ is embedded into $S_{n+k}$ as the subgroup that keeps $n+k$ fixed.

This representation, once restricted in the standard way to $S_{n}$, is not isomorphic to the natural $S_{n}$ representation ${ }^{3}$. Therefore it is also not compatible with the action on cohomology produced by the extended geometric $S_{n+1}$ action described in Section 4. Nevertheless it is interesting since it splits the cohomology module into a sum of induced representations of the form $\operatorname{In} d_{G}^{S_{n+k}} I d$ or $I n d_{G}^{S_{n+k-1}} I d$ where $G$ is the stabilizer of a partition of $\{1, \ldots ., n+k\}$.

Moreover, the orbits of this action can be used to write a generating formula for the Poincaré polynomials of the models $Y_{\mathcal{F}_{A_{n-1}}}$ that is different from the recursive formula for the Poincaré series recalled at the beginning of Section 8 .

Let us denote by $\Psi(q, t, z)$ the following exponential generating series:

$$
\Psi(q, t, z)=1+\sum_{n \geq 2, \mathcal{S} \in \mathcal{N}\left(\mathcal{F}_{A_{n-1}}\right)} P(S) z^{|\mathcal{S}|} \frac{t^{n+|\mathcal{S}|-1}}{(n+|\mathcal{S}|-1) !}
$$

where, for every $n \geq 2$,

- $\mathcal{S}$ ranges over all the nested sets of the building set $\mathcal{F}_{A_{n-1}}$ (i.e., $\mathcal{S}$ may not contain $\{V\})$;

- $P(\mathcal{S})$ is the polynomial, in the variable $q$, that expresses the contribution to $H^{*}\left(Y_{\mathcal{F}_{A_{n-1}}}, \mathbb{Z}\right)$ provided by all the monomials $m_{f}$ in the Yuzvinski basis such that supp $f=\mathcal{S}$. For instance, with reference to the Example 10.1, if $\mathcal{S}$ is the nested set $\left\{A_{1}, A_{2}\right\}$, then $P(\mathcal{S})=\left(q+q^{2}\right) q$ since we have to take into account all the possible ways to label the partition

$$
\{1,2,3,5\}\{4,6,7\}\{8,9\} \text {, }
$$

while if $\mathcal{S}$ is $\left\{A_{1}, V\right\}$ then $P(\mathcal{S})=\left(q+q^{2}\right)^{2}$ since we are dealing with the possible labellings of the partition

$$
\{1,2,3,5\}\{4,6,7,8\} \text {. }
$$

\footnotetext{
${ }^{3}$ One can see this for instance by counting the multiplicity of the trivial representation in $H^{6}\left(Y_{A_{7}}\right)$. The key point is provided by the monomials of type $c_{A_{1}}^{1} c_{A_{2}}^{1} c_{A_{3}}^{1} c_{V}^{0}$ that span an invariant subspace $H$ for both the natural $S_{8}$ action and the extended $S_{10}$ action. By an argument similar to that of Remark 9.1 i.e. by counting the number of orbits, one can check that on $H$ the natural $S_{8}$ representation and the $S_{8}$ representation restricted from $S_{10}$ differ in the the multiplicity of the trivial representation (that is respectively 3 and 4 ).
} 
We observe that the series $\Psi(q, t, z)$ encodes the same information that is encoded by the Poincaré series. In particular, for a fixed $n$, the Poincaré polynomial of the model $Y_{\mathcal{F}_{A_{n-1}}}$ can be read from the coefficients of the monomials whose $z, t$ component is $t^{k} z^{s}$ with $k-s=n-1$ (see the Example 10.2 at the end of this section).

Theorem 10.1. We have the following formula for the series $\Psi(q, t, z)$ :

$$
\Psi(q, t, z)=e^{t} \prod_{i \geq 3} e^{z q[i-2] q \frac{t^{i}}{i !}}
$$

where $[j]_{q}$ denotes the $q$-analog of $j:[j]_{q}=1+q+\cdots+q^{j-1}$.

Proof. We think of the monomials of the Yuzvinsky bases as labelled partitions. Then we single out the contribution given to $\Psi$ by all the parts represented by subsets with cardinality $i \geq 3$ and with non trivial label. If in a partition there is only one such part its contribution is $z\left(q+q^{2}+\cdots+q^{i-2}\right) \frac{t^{i}}{i !}$, if there are $j$ such parts their contribution is $z^{j}\left(q+q^{2}+\cdots+q^{i-2}\right)^{j} \frac{\left(\frac{t^{i}}{i j}\right)^{j}}{j !}$. In conclusion the contribution of all the parts represented by subsets with cardinality $i \geq 3$ and with non trivial label is provided by

$$
e^{z\left(q+q^{2}+\cdots+q^{i-2}\right) \frac{t^{i}}{i !}}-1
$$

Let us now focus on the contribution to $\Psi$ that comes from the parts with cardinality $i \geq 2$ and with label equal to 0 . For every monomial in the basis there is at most one such part, and its contribution is $\frac{t^{i-1}}{(i-1) !}$. The exponent $i-1$ (instead of $i$ ) takes into account that such part does not contribute to the cardinality $|\mathcal{S}|$. The total contribution of the elements with label equal to 0 is therefore $\sum_{i \geq 2} \frac{t^{i-1}}{(i-1) !}$. Summing up, we observe that the expression

$$
e^{t} \prod_{i \geq 3} e^{z q[i-2]_{q} \frac{t^{i}}{i !}}
$$

allows us to take into account the contribution to $\Psi$ of all the possible monomials in the Yuzvinski bases.

Example 10.2. If one wants to compute the Poincaré polynomial of $Y_{\mathcal{F}_{A_{4}}}$ one has to single out all the monomials in $\Psi$ whose $z, t$ component is $t^{k} z^{s}$ with $k-s=4$. A product of the exponential functions that appear in the formula (3) gives:

$$
\frac{t^{4}}{4 !}[1]+\frac{t^{5}}{5 !} z\left[16 q+6 q^{2}+q^{3}\right]+\frac{t^{6}}{6 !} z^{2}\left[10 q^{2}\right]
$$

Therefore the Poincaré polynomial is $1+16 q+16 q^{2}+q^{3}$.

\section{REFERENCES}

[1] Filippo Callegaro and Giovanni Gaiffi, The $S_{n+1}$ action on spherical models and supermaximal models of tipe $A_{n-1}, 2014$. in preparation.

[2] Francesco Cavazzani and Luca Moci, Geometric realizations and duality for DahmenMicchelli modules and De Concini-Procesi-Vergne modules, 2013. arXiv:1303.0902.

[3] Louis Comtet, Advanced combinatorics, enlarged, D. Reidel Publishing Co., Dordrecht, 1974. The art of finite and infinite expansions. MR0460128 (57 \#124)

[4] Corrado De Concini and Claudio Procesi, Wonderful models of subspace arrangements, Selecta Math. (N.S.) 1 (1995), no. 3, 459-494. MR1366622 (97k:14013) 
[5] _ Hyperplane arrangements and holonomy equations, Selecta Math. (N.S.) 1 (1995), no. 3, 495-535. MR1366623 (97k:14014)

[6] _ On the geometry of toric arrangements, Transform. Groups 10 (2005), no. 3-4, 387422. MR2183118 (2006m:32027)

[7] _ Topics in hyperplane arrangements, polytopes and box-splines, Universitext, Springer, New York, 2011. MR2722776 (2011m:52036)

[8] Emanuele Delucchi, Nested set complexes of Dowling lattices and complexes of Dowling trees, J. Algebraic Combin. 26 (2007), no. 4, 477-494. MR2341861 (2008i:05190)

[9] Graham Denham, Toric and tropical compactifications of hyperplane complements, 2013. arXiv:1306.3519.

[10] Vladimir G. Drinfel' $\mathrm{d}$, On quasitriangular quasi-Hopf algebras and on a group that is closely connected with $\operatorname{Gal}(\overline{\mathbb{Q}} / \mathbb{Q})$, Algebra i Analiz 2 (1990), no. 4, 149-181. MR1080203 (92f:16047)

[11] Pavel Etingof, André Henriques, Joel Kamnitzer, and Eric M. Rains, The cohomology ring of the real locus of the moduli space of stable curves of genus 0 with marked points, Ann. of Math. (2) 171 (2010), no. 2, 731-777. MR2630055 (2012a:14067)

[12] Eva Maria Feichtner, De Concini-Procesi wonderful arrangement models: a discrete geometer's point of view, Combinatorial and computational geometry, 2005, pp. 333-360. MR2178326 (2006i:05178)

[13] Eva Maria Feichtner and Dmitry N. Kozlov, Incidence combinatorics of resolutions, Selecta Math. (N.S.) 10 (2004), no. 1, 37-60. MR2061222 (2006k:06008)

[14] Eva Maria Feichtner and Bernd Sturmfels, Matroid polytopes, nested sets and Bergman fans, Port. Math. (N.S.) 62 (2005), no. 4, 437-468. MR2191630 (2006j:05036)

[15] Eva Maria Feichtner and Sergey Yuzvinsky, Chow rings of toric varieties defined by atomic lattices, Invent. Math. 155 (2004), no. 3, 515-536. MR2038195 (2004k:14009)

[16] William Fulton and Robert MacPherson, A compactification of configuration spaces, Ann. of Math. (2) 139 (1994), no. 1, 183-225. MR1259368 (95j:14002)

[17] Giovanni Gaiffi, Blowups and cohomology bases for De Concini-Procesi models of subspace arrangements, Selecta Math. (N.S.) 3 (1997), no. 3, 315-333. MR1481132 (99d:52009)

[18] _ Generalized Poincaré series for models of the braid arrangements, Manuscripta Math. 97 (1998), no. 3, 353-369. MR1654796 (2000j:14084)

[19] _ Models for real subspace arrangements and stratified manifolds, Int. Math. Res. Not. 12 (2003), 627-656. MR1951400 (2004d:52021)

[20] — Nested sets, set partitions and Kirkman-Cayley dissection numbers, 2014. arXiv: 1404.3395.

[21] Giovanni Gaiffi and Matteo Serventi, Poincaré series for maximal De Concini-Procesi models of root arrangements, Atti Accad. Naz. Lincei Cl. Sci. Fis. Mat. Natur. Rend. Lincei (9) Mat. Appl. 23 (2012), no. 1, 51-67. MR2924891

[22] _ Families of building sets and regular wonderful models, European J. Combin. 36 (2014), 17-38. MR3131872

[23] Ezra Getzler, Operads and moduli spaces of genus 0 Riemann surfaces, The moduli space of curves (Texel Island, 1994), 1995, pp. 199-230. MR1363058 (96k:18008)

[24] Yi Hu, A compactification of open varieties, Trans. Amer. Math. Soc. 355 (2003), no. 12, 4737-4753. MR1997581 (2004d:14080)

[25] Sean Keel, Intersection theory of moduli space of stable $n$-pointed curves of genus zero, Trans. Amer. Math. Soc. 330 (1992), no. 2, 545-574. MR1034665 (92f:14003)

[26] V. A. Krasnov, Real algebraically maximal varieties, Mat. Zametki 73 (2003), no. 6, 853-860; English transl., Math. Notes 73 (2003), no. 5-6, 806-812. MR2010655 (2004k:14111)

[27] Greg Kuperberg and Dylan P. Thurston, Perturbative 3-manifolds invariants by cut-andpaste topology, 1999. arXiv:GT/9912167.

[28] Pascal Lambrechts, Victor Turchin, and Ismar Volić, Associahedron, cyclohedron and permutohedron as compactifications of configuration spaces, Bull. Belg. Math. Soc. Simon Stevin 17 (2010), no. 2, 303-332. MR2663475 (2011d:51035)

[29] Li Li, Wonderful compactification of an arrangement of subvarieties, Michigan Math. J. 58 (2009), no. 2, 535-563. MR2595553 (2011f:14086)

[30] Robert MacPherson and Claudio Procesi, Making conical compactifications wonderful, Selecta Math. (N.S.) 4 (1998), no. 1, 125-139. MR1623714 (2001b:32032)

[31] Yu. I. Manin, Generating functions in algebraic geometry and sums over trees, The moduli space of curves (Texel Island, 1994), 1995, pp. 401-417. MR1363064 (97e:14065) 
[32] Zoran Petrić, On stretching the interval simplex-permutohedron, J. Algebraic Combin. 39 (2014), no. 1, 99-125. MR3144394

[33] Alex Postnikov, Victor Reiner, and Lauren Williams, Faces of generalized permutohedra, Doc. Math. 13 (2008), 207-273. MR2520477 (2010j:05425)

[34] Alexander Postnikov, Permutohedra, associahedra, and beyond, Int. Math. Res. Not. IMRN 6 (2009), 1026-1106. MR2487491 (2010g:05399)

[35] Eric M. Rains, The action of $S_{n}$ on the cohomology of $\bar{M}_{0, n}(\mathbb{R})$, Selecta Math. (N.S.) 15 (2009), no. 1, 171-188. MR2511203 (2010g:14035)

[36] _ The homology of real subspace arrangements, J. Topol. 3 (2010), no. 4, 786-818. MR2746338 (2012e:14109)

[37] Richard P. Stanley, A survey of alternating permutations, Combinatorics and graphs, 2010, pp. 165-196. MR2757798 (2012d:05015)

[38] Alexander P. Ulyanov, Polydiagonal compactification of configuration spaces, J. Algebraic Geom. 11 (2002), no. 1, 129-159. MR1865916 (2002j:14004)

[39] Michelle L. Wachs, Poset topology: tools and applications, Geometric combinatorics, 2007, pp. 497-615. MR2383132

[40] Sergey Yuzvinsky, Cohomology bases for the De Concini-Procesi models of hyperplane arrangements and sums over trees, Invent. Math. 127 (1997), no. 2, 319-335. MR1427621 (98m:14020) 